\title{
EMD Revisited: A New Understanding of the Envelope and Resolving the Mode-Mixing Problem in AM-FM Signals
}

\author{
Xiyuan Hu, Silong Peng and Wen-Liang Hwang ${ }^{\dagger}$
}

\begin{abstract}
Empirical Mode Decomposition (EMD) is an adaptive and data-driven approach for analyzing multicomponent nonlinear and non-stationary signals. The stop criterion, envelope technique, and mode-mixing problem are the most important topics that need to be addressed in order to improve the EMD algorithm. In this paper, we study the envelope technique and the mode-mixing problem caused by separating multicomponent AM-FM signals with the EMD algorithm. We present a new necessary condition on the envelope that questions the current assumption that the envelope passes through the extrema points of an intrinsic mode function (IMF). Then, we present a solution to the mode-mixing problem that occurs when multicomponent AM-FM signals are separated. We experiment on several signals, including simulated signals and real-life signals, to demonstrate the efficacy of the proposed method in resolving the modemixing problem.
\end{abstract}

\section{INTRODUCTION}

Single-channel signal separation and estimation has attracted a great deal of attention in recent years because it affects many applications. Typical single-channel signal separation approaches model a signal

This work was supported by the National Natural Science Foundation of China (60972126), the Joint Funds of the National Natural Science Foundation of China (U0935002/L05), the Beijing Natural Science Foundation (4102060), the State Key Program of National Natural Science Foundation of China (Grant No.61032007), and the National Science Council of Taiwan (Grant No. 100-2221-E-001-017-).

Xiyuan Hu and Silong Peng are with the Institute of Automation, The Chinese Academy of Sciences, Beijing 100190, China (e-mail:\{xiyuan.hu,silong.peng\}@ia.ac.cn).

Wen-Liang Hwang is with Institute of Information Science, Academia Sinica, Taipei 11529, Taiwan and Department of Information Management, Kainan University, Taiwan. (e-mail:whwang@iis.sinica.edu.tw). 
as a superposition of additive coherent basic signals. For instance, a nonlinear and non-stationary signal can be modeled as a multicomponent AM-FM signal. The methods used to separate signals vary because different subcomponents are used to construct the signals. Recently, the Empirical Mode Decomposition (EMD) approach has generated a lot of interest because it has a number of useful features [1].

The EMD algorithm is a fully data-driven and self-adaptive process that models the target signal as a series of intrinsic mode functions (IMFs) plus a residual signal. An IMF satisfies two conditions: (1) in the whole data set, the number of extrema and the number of zero-crossings must be equal, or differ by one at most and (2) at any point, the local average of the upper and lower envelopes must be zero. To obtain an IMF, the EMD uses a sifting process based on the estimated upper and lower envelopes, interpolated from the extrema, of the input signal. The theoretical analysis and experiments on envelope interpolation are discussed in [2], [3]. However, because of the obscure nature of the envelope, mathematically explicit and physically meaningful answers to questions like "What is an envelope?" and "What constitutes a good envelope?" are still elusive. We do not provide a definition of the envelope in this paper; however, we derive the necessary conditions that an envelope must satisfy in EMD.

EMD extracts the highest frequency component locally as the current IMF, which is derived from the extrema and the envelopes during the sifting process. Hence, it is inevitable that the IMF will be affected by the mode mixing problem caused by the intermittency, as noted by Wu and Huang [4]. The intermittency was originally referred to as the alternation of phases of apparently periodic and chaotic dynamics in turbulence. In this paper, we use the definition in [5], where the intermittency is referred to as a component that comes into existence or disappears from a signal entirely at a particular time scale. The mode mixing problem occurs when the frequency tracks of an IMF jump as an intermittent component arrives or departs.

In this paper, we analyze the properties of the envelope and the mode-mixing problem caused by separating AM-FM signals with the EMD algorithm. First, we analyze the properties of the envelope. Then, we propose an AM-FM demodulation algorithm, which is used later to solve the mode-mixing problem of an IMF.

Normally, a multicomponent AM-FM signal is used to model a non-stationary signal and applied in the analysis of a broad range of signals in various fields, such as mechanics and vibrations [4], [6]. The IMF of a signal is always a monocomponent AM-FM signal, which facilitates meaningful instantaneous frequency (IF) estimation via the Hilbert transform, Teager-Kaiser energy operator [7] or other techniques [8], [9], [10], [11]. We study the envelope of a monocomponent AM-FM signal $a(t) \cos (\phi(t))$ and observe that its upper (resp. lower) envelope does not necessarily pass through its maximum (resp. minimum) points; 
however, it does pass by, and is tangential to, the points with a phase angle $\phi(t)$ of $2 k \pi((2 k+1) \pi)$. This observation motivates us to present a new necessary condition for the envelopes of an IMF as well as a numerical algorithm to estimate the envelopes. We also propose an optimization approach to demodulate the AM and FM parts of the IMF from the estimated envelopes.

The mode mixing problem of sine waves has been studied and resolved by Deering and Kaiser [5]. For a signal $a_{1} \sin \left(2 \pi f_{1} t\right)+a_{2} \sin \left(2 \pi f_{2} t\right)$, the mode mixing problem occurs when some values on $a_{1}$, $a_{2}, f_{1}$ and $f_{2}$ are chosen. A detailed analysis of the parameter values used to generate the mode-mixing phenomenon of the EMD for two sine waves can be found in [12]. For example, if $a_{1}=a_{2}$ and $0.5<f_{1} / f_{2}<2[3]$, then the first IMF of the signal is a modulated signal that contains the higher frequency component and a portion of the lower frequency component. Basically, Deering and Kaiser's solution involves inserting a masking sine signal that prevents the lower frequency component from being included in the IMF. Their approach is interesting and innovative; however, it cannot resolve the mode mixing problem in AM-FM modulated signals. Therefore, to address the problem, we propose a new method that is a generalization of Deering and Kaiser's algorithm. We derive the conditions for our method to achieve the goal and demonstrate the efficacy of the proposed algorithm on simulated and real-life signals.

The remainder of this paper is organized as follows. In Section II, we review the EMD algorithm and some related works on the envelope and mode mixing problem. In Section III, we propose a new necessary condition for an IMF's envelope, as well as a numerical algorithm to derive an envelope that satisfies the proposed condition. We also present an AM and FM demodulation algorithm based on the derived envelope. In Section IV, we discuss the mode mixing phenomenon in AM-FM signals and propose an algorithm to resolve the problem. We also present the results of experiments on simulated and real-life signals. Section V contains some concluding remarks.

\section{EMD PRELIMINARIES AND DISCUSSION}

The objective of the EMD method is to identify IMFs by using a sifting process. Given a real input signal $s(t)$, let $r(t)=s(t), k=1$, and $i=0$. The steps of the sifting process are as follows.

1) Find all local minima and maxima of $r(t)$.

2) Interpolate between the minima (resp., maxima) to acquire the lower envelope $e_{\min }(t)$ (resp., the upper envelope $\left.e_{\max }(t)\right)$.

3) Compute the mean envelope as an approximation of the local average $m(t)=\left(e_{\min }(t)+e_{\max }(t)\right) / 2$.

4) Let $i=i+1$ and define the proto-mode function (PMF) as $p_{i}(t)=r(t)-m(t)$, and let $r(t)=p_{i}(t)$. 
5) Repeat Steps 1) to 4) on PMF $p_{i}(t)$ until it becomes an IMF; then, record the $\operatorname{IMF} \operatorname{imf}_{k}(t)=p_{i}(t)$.

6) Let $r(t)=r(t)-\operatorname{imf}_{k}(t)$. If the number of extrema of $r(t)$ is greater than three, let $k=k+1$, $i=0$, and go to Step 1; otherwise, stop the sifting process.

Steps 3 and 5 compute the mean envelope and test the stopping criterion of an IMF respectively. Different implementations of the two steps yield different decomposition results. For details of the EMD algorithm and its implementation, readers may refer to [1] and [3]. The algorithm represents $s(t)$ as the summation of IMFs and a residual $r(t)$ as follows:

$$
s(t)=\sum_{k} \operatorname{imf}_{k}(t)+r(t)
$$

\section{A. The envelope algorithm}

Computing a good mean envelope $m(t)$ and testing the stop criterion of an IMF are crucial to the success of the EMD algorithm. Clearly, the upper and lower envelopes play a significant role in both of these steps. In the standard EMD algorithm [1], after identifying the extrema, a cubic spline-based interpolation method is used to generate the upper and lower envelopes. Several interpolation methods have been proposed to improve the performance of the standard EMD algorithm. For example, the B-spline and some higher order polynomial interpolation methods are used to replace the cubic spline interpolation technique [2], [13]. Meanwhile, because of the drawbacks of using extrema, many algorithms designed for computing the mean envelope directly, such as the PDE method [14], the Genetic Algorithm [15] and the Local Integral Mean method [10], [16], have been applied to the sifting process. In the image analysis area, some envelope approaches, such as the radial basis function and optimization, are used as two-dimensional (2-D) extensions of the EMD algorithm [17], [18], [19], [20]. Although the cited methods improved on the single channel separation performance of the standard EMD algorithm, the envelopes are still defined by their algorithms and the criteria for an envelope are still not completely understood.

\section{B. The mode-mixing problem}

Mode mixing is another major problem when EMD is used to decompose signals. According to [21], there are two possible causes of the problem: 1) the IMF contains signals of widely disparate scales; or 2) signals of a similar scale reside in different IMF components. To decompose $s(t)=s_{h}(t)+s_{l}(t)$ by the EMD, where $s_{h}(t)$ and $s_{l}(t)$ denote the high frequency component and the low frequency component respectively, the problem occurs when parts of the components of $s_{h}(t)$ are completely immersed in 
the corresponding parts of $s_{l}(t)$. Thus, the sifting process detects the extrema of $s_{l}(t)$ in the parts of $s_{h}(t)$ that are immersed. However, the sifting process also detects the extrema of $s_{h}(t)$ in other parts of the signal $s(t)$. This phenomenon can create intermittency in the extrema of the signal; that is, the extrema detected in the sifting process belong to different signals. As a consequence, the IMF is a signal comprised of different scales; some parts are from signal $s_{h}$ and the other parts are from signal $s_{l}$.

Several methods have been proposed to solve the mode-mixing problem under EMD. In [4], an intermittency test is used to provide a choice of extrema for the envelopes in the sifting process. However, for complicated data with variable scales, this approach is rather difficult because some single intermittency test criteria are not applicable. Deering and Kaiser [5] adopted a pure sine wave as a masking signal to solve the mode-mixing problem of an IMF of the form $a_{1} \sin \left(2 \pi f_{1} t\right)+a_{2} \sin \left(2 \pi f_{2} t\right)$. The masking signal is a high frequency sine wave that is added to the input signal $s(t)$ to make the extrema of the immersed parts of the high frequency component detectable in the sifting process. The steps of the algorithm are as follows.

1) Construct a masking signal, $\hat{m}(t)$.

2) Perform EMD on $s_{+}(t)=s(t)+\gamma \hat{m}(t)$ to obtain the IMF $h_{+}(t)$.

3) Perform EMD on $s_{-}(t)=s(t)-\gamma \hat{m}(t)$ to obtain the IMF $h_{-}(t)$.

4) Derive the IMF of $s(t)$ as $z(t)=\left(h_{+}(t)+h_{-}(t)\right) / 2$.

The parameter $\gamma$ is multiplied by the masking signal $\hat{m}(t)$ to enlarge the amplitude of the high frequency component so that performing EMD on $s_{+}(t)$ and $s_{-}(t)$ can extract the high frequency component of $s(t)$. This approach can only be used to resolve the mode-mixing problem of two sine waves, and the success of the algorithm depends on the accuracy of the estimated high frequency component of the input signal. The recently proposed ensemble EMD (EEMD) algorithm [21] uses a large number of noisy signals as masking signals. It is shown that EEMD can resolve the mode-mixing problem in some real-life signals. However, they did not provide an analysis on the feasibility of using the approach to solve the mode-mixing problem.

\section{A New View of the Envelope Technique}

We model the input signal $s(t)$ as a multicomponent AM-FM signal in which

$$
s(t)=\sum_{i=1}^{M} s_{i}(t)=\sum_{i=1}^{M} a_{i}(t) \cos \left(\phi_{i}(t)\right) \text { with } a_{i}(t)>0 .
$$

Each mono-component AM-FM signal $s_{i}(t)$ can be regarded as an IMF. The rationale is that the FM part, $\cos \left(\phi_{i}(t)\right)$, and the AM part (with $a_{i}(t)$ as the upper envelope and $-a_{i}(t)$ as the lower envelope), 
of $s_{i}(t)$ satisfy the first and second conditions of an IMF respectively. In this section, we first propose a new necessary condition on the envelope for a mono-component AM-FM signal to be an IMF; then, we present a numerical algorithm to estimate the envelope.

\section{A. Envelope for AM-FM signals}

Most of the works related to the envelope of the sifting process are based on the two principles proposed by Huang et al. in [1]:

- The envelope must be continuous and must pass through the extrema (maxima and minima).

- The first derivative of the envelope must be continuous.

Based on these principles, the process for computing a specific signal's upper (resp. lower) envelope involves two steps: 1)identify the maximum (resp. minimum) points of the signal; and then 2) use a smooth interpolation function to interpolate between the maximum (resp. minimum) points. Unfortunately, if we consider the amplitude part $a(t)$ of a mono-component AM-FM signal $s(t)=a(t) \cos (\phi(t))$ as its envelope, in most cases, the envelope $a(t)$ will not pass through the maximum (and minimum) points of the signal $s(t)$.

For example, if we let $t_{0}$ be a point in the upper envelope of $s(t)$, we have

$$
a\left(t_{0}\right)=s\left(t_{0}\right)=a\left(t_{0}\right) \cos \left(\phi\left(t_{0}\right)\right)
$$

If $t_{0}$ is also a maximum point of $s(t)$, we have

$$
s^{\prime}\left(t_{0}\right)=a^{\prime}\left(t_{0}\right) \cos \left(\phi\left(t_{0}\right)\right)-a\left(t_{0}\right) \sin \left(\phi\left(t_{0}\right)\right) \phi^{\prime}\left(t_{0}\right)=0,
$$

where the prime denotes the first order derivative with respect to $t$. From Equations (3) and (4), we can derive $\cos \left(\phi\left(t_{0}\right)\right)=1$ and $a^{\prime}\left(t_{0}\right)=0$. This indicates that there is a correlation between the amplitude part $a(t)$ and the phase part $\phi(t)$ at the maximum points. Because the amplitude $a(t)$ and phase $\phi(t)$ of $a(t) \cos (\phi(t))$ may be completely uncorrelated, we conclude that the envelope $a(t)$ of the signal does not necessarily pass through the extrema of $s(t)$, as shown in Figure 1.

Based on the above analysis and our observations, we modify the first principle of the envelope proposed by Huang et al. in [1] and present a new necessary condition on the envelope of an AM-FM signal $s(t)=a(t) \cos (\phi(t))$ : The upper (lower) envelope should pass through and be tangential to the signal at all the points that satisfy $\phi(t)=2 k \pi((2 k+1) \pi)$. This condition uses the value of the signal and also exploits the information about the phase angle of a frequency modulated function. Moreover, it conforms to the intuition that an envelope of an AM-FM signal should be on and tangential to the signal. 
For convenience, we denote the points in $s(t)$ that satisfy the above necessary condition as tangential points. Let the upper envelope of the signal $s(t)$ be $\psi_{u}(t)$. This necessary condition requires that, if $\phi\left(\tau_{u, i}\right)=2 k \pi$ at the points $\tau_{u, i}$, then the the upper envelope $\psi_{u}$ must satisfy

$$
s\left(\tau_{u, i}\right)=a\left(\tau_{u, i}\right) \cos \left(\phi\left(\tau_{u, i}\right)\right)=a\left(\tau_{u, i}\right)=\psi_{\mathbf{u}}\left(\tau_{u, i}\right),
$$

and

$$
s^{\prime}\left(\tau_{u, i}\right)=a^{\prime}\left(\tau_{u, i}\right) \cos \left(\phi\left(\tau_{u, i}\right)\right)-a\left(\tau_{u, i}\right) \sin \left(\phi\left(\tau_{u, i}\right)\right) \phi^{\prime}\left(\tau_{u, i}\right)=a^{\prime}\left(\tau_{u, i}\right)=\psi_{\mathrm{u}}^{\prime}\left(\tau_{u, i}\right) .
$$

Similarly, we can draw the same conclusion about the lower envelope.

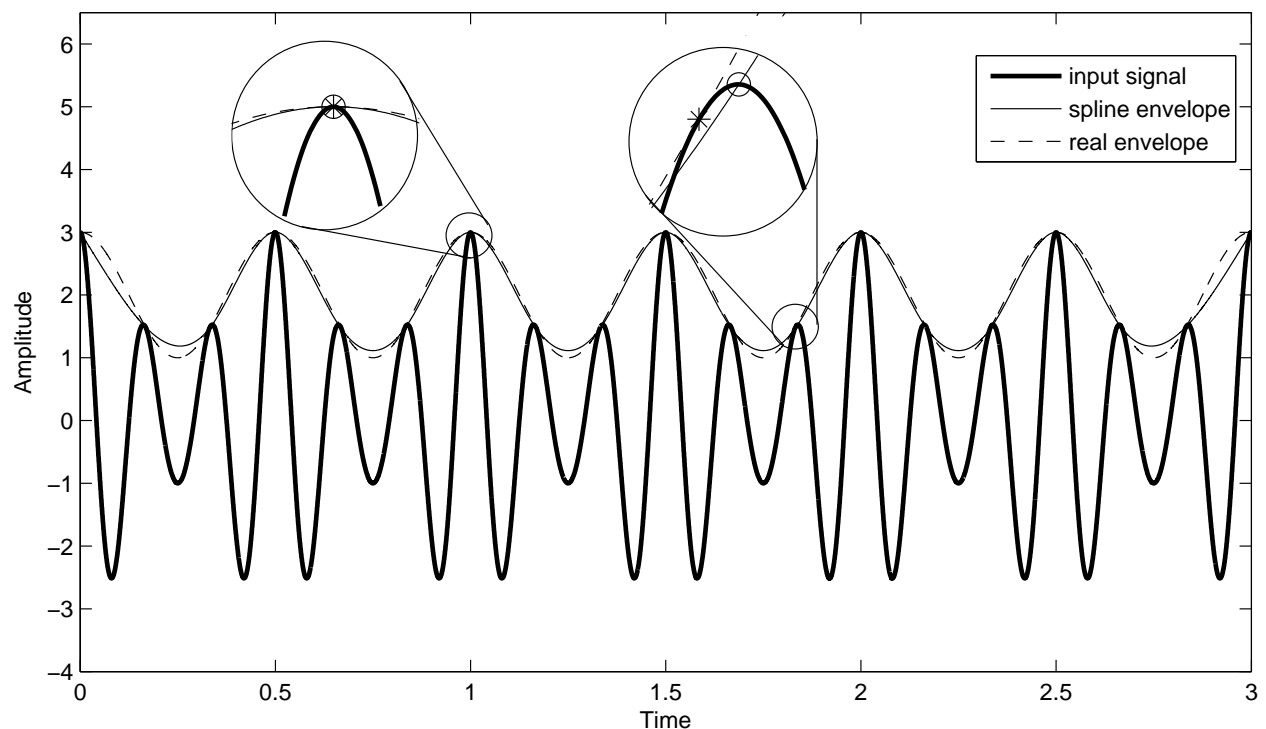

Fig. 1. Ground truth upper envelope (dashed curve) and the constructed upper envelope acquired through cubic spline interpolation (thin curve) of the signal $s(t)=(2+\cos (2 \pi t)) \cos (6 \pi t)$ (thick curve). The stars and circles denote the tangential points and local maximum points respectively. From the interpolation points and the estimated envelope, we observe that the envelope passes by, and is tangential to, the signal at the local maximum points, where the first derivative of the envelope is equal to zero. However, the envelope does not pass through the other local maxima, nor is it tangential to them. In other words, the envelope will pass through some points (including local extrema points) of the signal, and the first derivative of the envelope will coincide with that of the signal at those points.

\section{B. Numerical computation of the envelope}

Next, we introduce a numerical approximation algorithm for computing the envelope of a discrete AM-FM signal using the necessary condition defined above. Since the condition is similar for the upper and the lower envelopes, we only discuss the algorithm for estimating the upper envelope. 
For an upper envelope to satisfy the necessary condition, it must pass through the tangential points, defined as the points of signal $s(t)=a(t) \cos (\phi(t))$, where the phase angles of $\cos (\phi(t))$ are $2 k \pi$. It is quite difficult to find the tangential points in $s(t)$ because the FM function $\cos (\phi(t))$ has been modulated by an AM function $a(t)$. However, if we assume that the AM function varies much more slowly than the FM function, the tangential points are close to the maximum points of $s(t)$. Therefore, we can locate the local maximum points, and use the Taylor expansion to compute the offset distance between the tangential points and the local maximum points. Let $t_{i}$ denote the position of $i$ th local maxima. For each $t_{i}$, we search for the offset $\Delta t_{i}$ that conforms to the following equations:

$$
\begin{gathered}
\psi\left(t_{i}+\Delta t_{i}\right)=s\left(t_{i}+\Delta t_{i}\right), \\
\psi^{\prime}\left(t_{i}+\Delta t_{i}\right)=s^{\prime}\left(t_{i}+\Delta t_{i}\right),
\end{gathered}
$$

where $\psi$ is the upper envelope to be estimated. When $\Delta t_{i}$ has been determined, the tangential points are at $t_{i}+\Delta t_{i}$. Based on the values of $\psi\left(t_{i}+\Delta t_{i}\right)$ and $\psi^{\prime}\left(t_{i}+\Delta t_{i}\right)$ at the tangential points, we use an interpolation method to derive the upper envelope. Spline interpolation can satisfy Equation (7), but not Equation (8). Thus, we use the Hermite polynomial method [22] to derive the upper envelope because it interpolates a polynomial that matches both the value and the first derivative of $s(t)$ at the tangential points.

Since it is hard to find the correct values of $\Delta t_{i}$ and the derivatives of $\psi(t)$ at $t_{i}+\Delta t_{i}$ in one step, we introduce an alternative optimization approach to calculate the offset $\Delta t_{i}$ and the envelope. The approach involves two steps: estimating the offset $\Delta t_{i}$, and updating the envelope $\psi(t)$. Let us suppose that the tangential points $t_{i}^{(k)}$ and the envelope $\psi^{(k)}$ have been established at the $k$ th iteration. We use the first order Taylor expansion in (8) to obtain

$$
\psi^{(k)^{\prime}}\left(t_{i}^{(k)}\right)+\psi^{(k)^{\prime \prime}}\left(t_{i}^{(k)}\right) \Delta t_{i}^{(k)}=s^{\prime}\left(t_{i}^{(k)}\right)+s^{\prime \prime}\left(t_{i}^{(k)}\right) \Delta t_{i}^{(k)},
$$

which yields

$$
\Delta t_{i}^{(k)}=\frac{s^{\prime}\left(t_{i}^{(k)}\right)-\psi^{(k)^{\prime}}\left(t_{i}^{(k)}\right)}{\psi^{(k)^{\prime \prime}}\left(t_{i}^{(k)}\right)-s^{\prime \prime}\left(t_{i}^{(k)}\right)},
$$

where the prime and the double prime denote, respectively, the first and second order derivatives with respect to $t$. In the discrete case, we use a numerical method to compute the first and second order derivatives of the signal and the envelope. Let $u[n]$ be a discrete signal. The values of $u^{\prime}[n]$ and $u^{\prime \prime}[n]$ are obtained by calculating $u^{\prime}[n]=(u[n+1]-u[n-1]) / 2$ and $u^{\prime \prime}[n]=u[n+1]+u[n-1]-2 u[n]$. 
Then, we update the tangential points by setting $t_{i}^{(k+1)}=t_{i}^{(k)}+\Delta t_{i}^{(k)}$. To update the envelope $\psi^{(k)}(t)$, we assume that

$$
\psi^{(k+1)}\left(t_{i}^{(k+1)}\right)=s\left(t_{i}^{(k+1)}\right)
$$

and approximate $\psi^{(k+1)^{\prime}}\left(t_{i}^{(k+1)}\right)$ by using

$$
\psi^{(k+1)^{\prime}}\left(t_{i}^{(k+1)}\right)=\frac{\psi^{(k+1)}\left(t_{i}^{(k)}\right)-\psi^{(k+1)}\left(t_{i}^{(k+1)}\right)}{-\Delta t_{i}^{(k)}} .
$$

In (12), we need to calculate the values of $\psi^{(k+1)}\left(t_{i}^{(k)}\right)$, which can be estimated by the second order Taylor expansion in (7) as follows:

$$
\psi^{(k+1)}\left(t_{i}^{(k)}\right)=s\left(t_{i}^{(k)}\right)+\left(s^{\prime}\left(t_{i}^{(k)}\right)-\psi^{(k)^{\prime}}\left(t_{i}^{(k)}\right)\right) \Delta t_{i}^{(k)}+0.5\left(s^{\prime \prime}\left(t_{i}^{(k)}\right)-\psi^{(k)^{\prime \prime}}\left(t_{i}^{(k)}\right)\right)\left(\Delta t_{i}^{(k)}\right)^{2} .
$$

From (11) and (12), we have the values of $\psi^{(k+1)}(t)$ and $\psi^{(k+1)^{\prime}}(t)$ at all the knots $t_{i}^{(k+1)}$. From the values and their derivatives on the knots, the upper envelope $\psi^{(k+1)}(t)$ can be derived by using the Hermite interpolation algorithm.

The two steps discussed above estimate the offset $\Delta t_{i}$ and the upper envelope $\psi(t)$ alternately. The algorithm stops when the values of the offset $\left|\Delta t_{i}\right|$ are close to zero or the maximum number of iterations have been performed. The procedure is summarized in Algorithm 1, and the results are presented in Figure 2. The right-hand column of the figure plots the curves of the maximum absolute difference of $\left|\Delta t_{i}^{(k)}\right|$ versus the number of iterations $k$ for the AM-FM signals in the same row of the left-hand column. The curves decrease and converge to some small number as the number of iterations increases.

\section{Demodulation of an AM-FM signal}

Here, we demonstrate that a simple optimization algorithm can be applied to the estimated envelope of an AM-FM signal to demodulate the AM and FM parts of the signal. Let $\hat{a}(t)$ denote the estimated envelope of the input signal $s(t)=a(t) \cos (\phi(t))$ by Algorithm 1. We can use a piecewise polynomial $\hat{\phi}\left(t ; \Theta_{p}\right)$, where $\Theta_{p}$ are the coefficients of the polynomial, to derive $\phi(t)$ by solving the following optimization problem:

$$
\begin{gathered}
\hat{\Theta}_{p}=\arg \min _{\hat{\phi}\left(t ; \Theta_{p}\right)}\left\{\left\|\hat{\phi}\left(t ; \Theta_{p}\right)-\arccos (s(t) / \hat{a}(t))\right\|^{2}\right\} \\
\text { for each } t_{i} \text { with }\left\{\begin{array}{c}
\hat{\phi}\left(t_{i} ; \Theta_{p}\right)=k \pi \\
\hat{\phi}_{i-1}^{(j)}\left(t_{i} ; \Theta_{p}\right)=\hat{\phi}_{i}^{(j)}\left(t_{i} ; \Theta_{p}\right), 1 \leq j \leq 2,
\end{array}\right.
\end{gathered}
$$



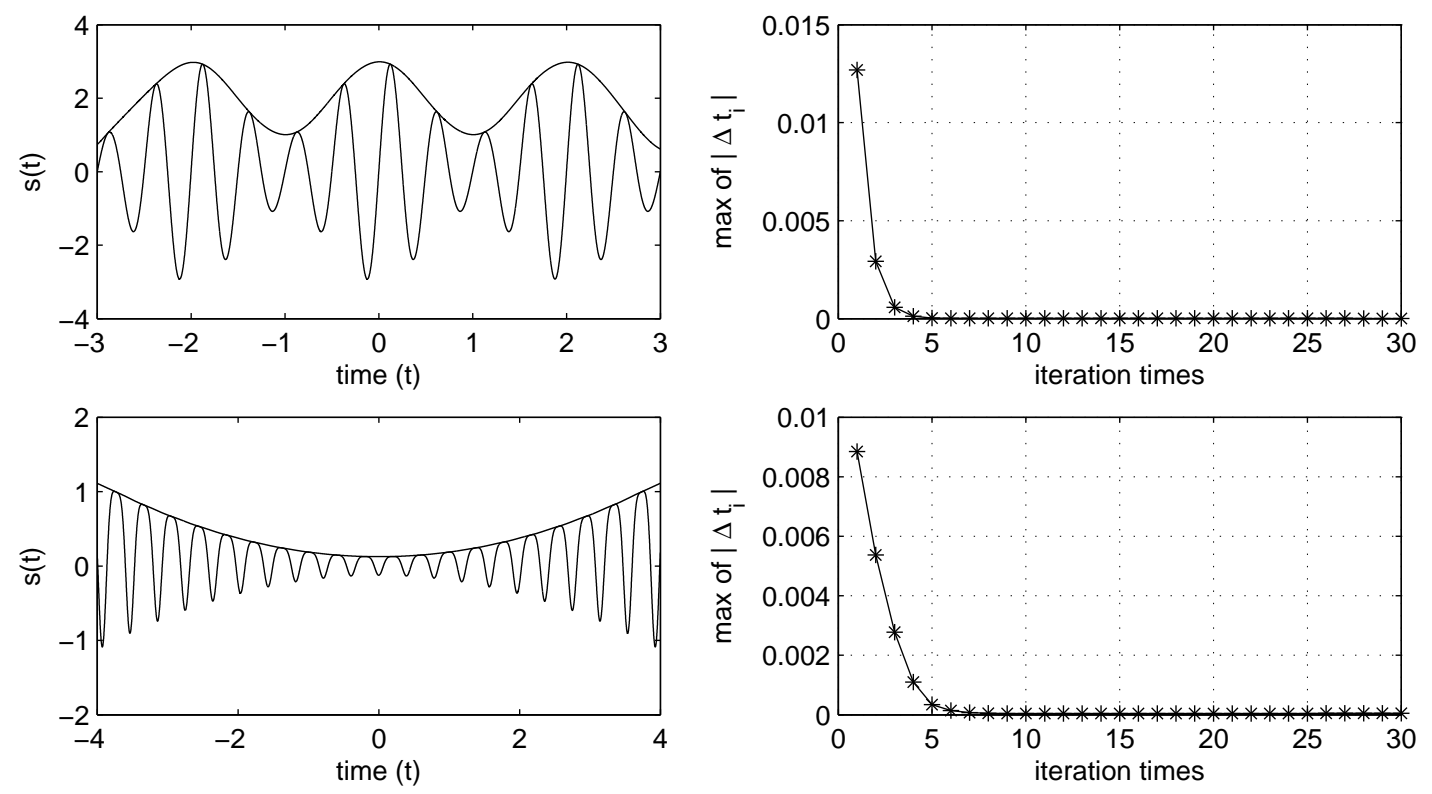

Fig. 2. The estimation procedure in Algorithm 1. Left-hand column: the input signals (top: $(2+\cos (\pi t)) \cos (4 \pi t+3 \pi / 2)$; bottom: $\left.\left(t^{2}+2\right) \cos (\pi \sin (8 t)+\pi) / 16\right)$ and the (upper) envelopes derived by the algorithm. Right-hand column: the plots of the maximum absolute difference of $\left|\Delta t_{i}^{(k)}\right|$ versus the number of iterations $k$ of the signals in the same row of the left-hand column.

where $t_{i}$ are the tangential points of $|s(t)| ; \hat{\phi}_{i}\left(t ; \Theta_{p}\right)$ denotes the segment of the estimated phase function from $t_{i}$ to $t_{i+1}$; and $\hat{\phi}_{i}^{(j)}\left(t_{i} ; \Theta_{p}\right)$ denotes the $j$ th derivatives of $\hat{\phi}_{i}\left(t ; \Theta_{p}\right)$ at $t_{i}$. Note that we do not use the Hilbert transform to estimate the phase because the approach cannot satisfy the constraints in Equation (16).

Figure 3 shows the results of our demodulation algorithm on a simulated signal. The mono-component AM-FM input signal is $s(t)=\left(t^{2}+2\right) \cos (\pi \sin (8 t)) / 16$, which is taken from [24]. Note that the phase of the signal is $\pi \sin (8 t)$. If we take the derivative of the phase function with respect to $t$ as the instantaneous frequency (IF), the derived IF may be negative. Thus, we replace the phase function with

$$
\pi\left((-1)^{\lceil(8 t+3 \pi / 2 \pi)\rceil} \sin (8 t)+2(\lceil(8 t+3 \pi / 2 \pi)\rceil-1)\right) .
$$

The values of the resulting signal are unchanged and the IF of the signal does not have negative values. The symbol $\lceil x\rceil$ denotes the operation that rounds the element $x$ to the nearest integers greater than or equal to $x$. The input signal $s(t)$ and its IF are shown in Figures 3(a) and (b) respectively. Figure 3(c) compares the estimated IF of our method and that of the Normalized Directly Compute Quadrature (NDQ) algorithm [23]. The errors with respect to the true IF of different methods are plotted in Figure 
(a)

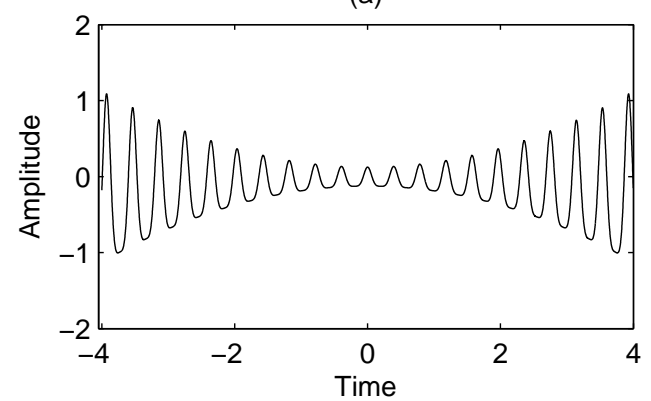

(c)

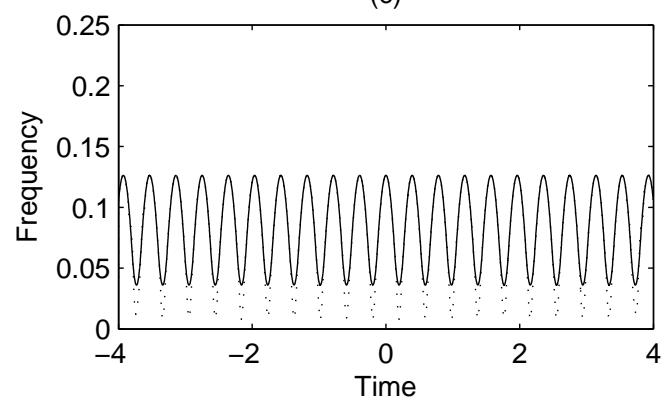

(b)

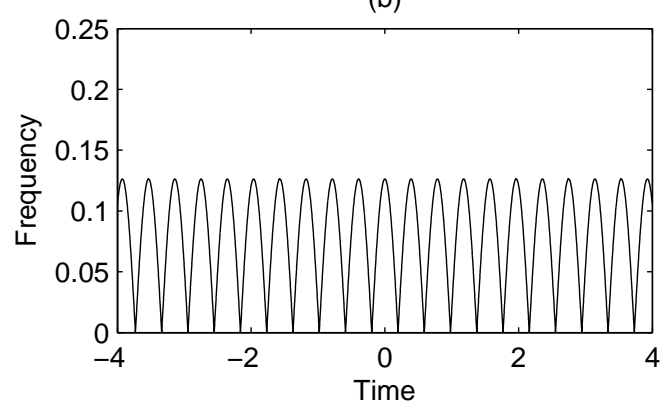

(d)

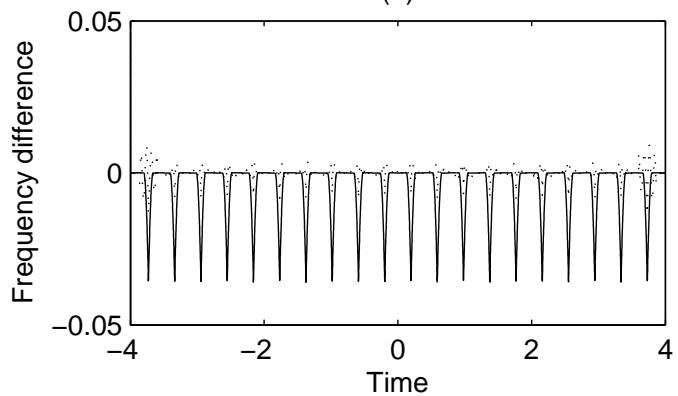

Fig. 3. AM-FM decomposition of a mono-component signal $s(t)=\left(t^{2}+2\right) \cos (\pi \sin (8 t)) / 16$ with $-4 \leq t \leq 4$ by our algorithm and the Normalized Direct Compute Quadrature (NDQ) algorithm [23]: (a) the input mono-component AM-FM signal; (b) the IF, which is normalized between 0 and 0.5 ; (c) the estimated IF of our algorithm and the NDQ approach, plotted by dotted and solid lines respectively; (d) the differences between the estimated instantaneous frequency of our approach and that of NDQ with the real IF, indicated by the dotted and solid lines respectively.

3(d). Note that the result derived by the NDQ algorithm does not necessarily satisfy the constraints in Equation (16).

\section{RESOLVING THE MODE-MIXING PROBLEM OF AM-FM SIGNALS}

In Section IV-C, we describe how the mode-mixing problem of sine waves can be solved by Deering and Kaiser's approach [5]. However, the method cannot be used to solve the mode-mixing problem of AM-FM signals. In many physical systems, such as complicated vibration systems with damping [25] and communication systems with non-linear modulation functions, the signals can be modeled as AMFM functions. Recall that two possible causes of the mode-mixing problem under EMD were mentioned in Section I. Figure 4 shows an example of mode-mixing in AM-FM signals. The input signal and its two subcomponents are shown in Figures 4(a), (b), and (c) respectively. The first IMF extracted by the EMD is shown in Figure 4(d). Note that the four segments, $S_{1}$ to $S_{4}$, in the IMF are low frequency FM 
components from the subcomponent in Figure 4(b); while the other parts of the IMF are high frequency FM components from the subcomponents in Figure 4(c). Obviously, the IMF is affected by the mode mixing-problem because it contains segments of different scales from different signals. In segments $S_{1}$ to $S_{4}$, the high frequency component is completely immersed in the low frequency component; thus, the EMD algorithm only detected the extrema of the low frequency component. Our approach is a generalization of Deering and Kaiser's approach, which we use to create a high frequency FM signal that can mask the extrema of the low frequency FM component in segments $S_{1}$ to $S_{4}$ during the EMD sifting process.

Our approach is comprised of three steps. First, we recover the high frequency information hidden in the extracted IMF; second, we construct a masking signal and use it to recover the desired IMF. We propose an algorithm to resolve the mode-mixing problem of two additive AM-FM signals and present a detailed discussion and analysis of the three steps of our algorithm in subsections A, B, and C respectively. The algorithm can be extended to solve the mode-mixing problem of more than two additive AM-FM signals.

In our model, the input signal is defined as

$$
s(t)=s_{l}(t)+s_{h}(t)=a_{l}(t) \cos \left(\phi_{l}(t)\right)+a_{h}(t) \cos \left(\phi_{h}(t)\right),
$$

where $a_{l}(t), a_{h}(t)>0$ and $\phi_{h}^{\prime}(t)>\phi_{l}^{\prime}(t)$; that is, $s_{l}(t)$ and $s_{h}(t)$ are the low frequency and high frequency FM components respectively. The condition $a_{l}(t), a_{h}(t)>0$ precludes the signals containing segments with zero values. We assume that the first IMF $g(t)$ of the $s(t)$ has the following form:

$$
g(t)=\tilde{a}_{l}(t) \cos \left(\tilde{\phi}_{l}(t)\right)+\tilde{a}_{h}(t) \cos \left(\tilde{\phi}_{h}(t)\right)
$$

where $\tilde{\phi}_{l}^{\prime}(t) \approx \phi_{l}^{\prime}(t)$ and $\tilde{\phi}_{h}^{\prime}(t) \approx \phi_{h}^{\prime}(t)$, and $\tilde{a}_{h}(t), \tilde{a}_{l}(t) \geq 0$. The mode-mixing problem of an IMF implies that some segments of $g(t)$ contain information from the low frequency FM signal $s_{l}(t)$ and the other segments contain the high frequency FM signal $s_{h}(t)$.

\section{A. Recovering the hidden high frequency component in an IMF}

First, we use an AM-FM signal $\hat{g}(t)$ to approximate the IMF $g(t)$. Then, we analyze the difference between signals $g(t)$ and $\hat{g}(t)$, denoted as $d(t)=g(t)-\hat{g}(t)$. We partition the domain of the IMF $g(t)$ into low frequency components and high frequency components. Let $\mathbf{S}$ denote the union of the low frequency segments (e.g. $\mathbf{S}$ is the union of $S_{1}$ and $S_{4}$ in Figure 4(d)), and let $\overline{\mathbf{S}}$ denote the high frequency segments. In addition, we assume that the end points of the segments in $\mathbf{S}$ and $\overline{\mathbf{S}}$ of $g(t)$ are not extrema points. 
(a)

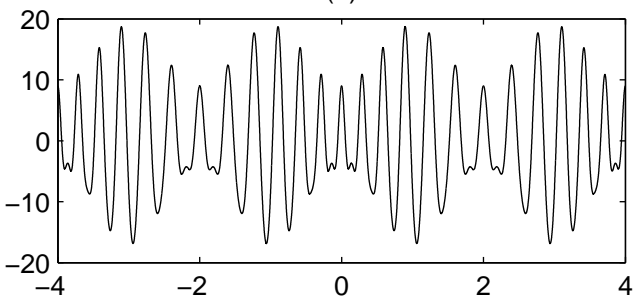

(c)

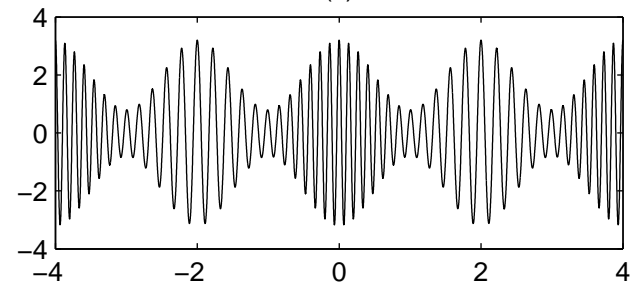

(e)

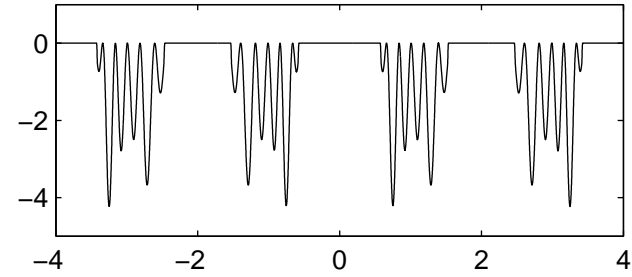

(g)

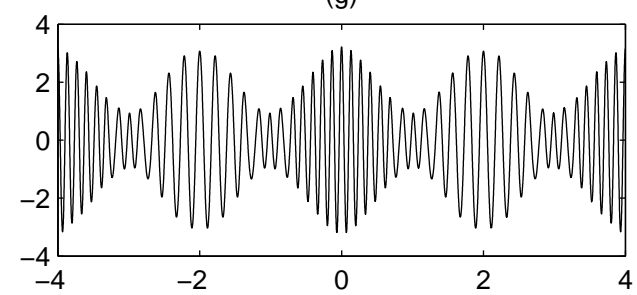

(b)

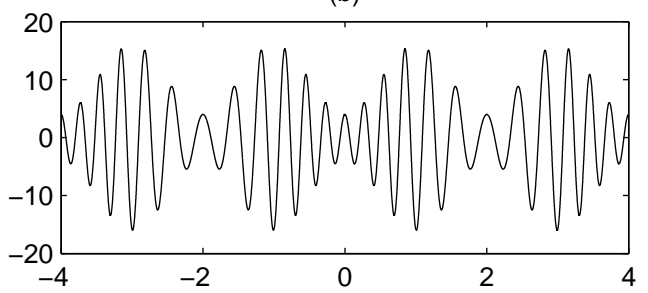

(d)

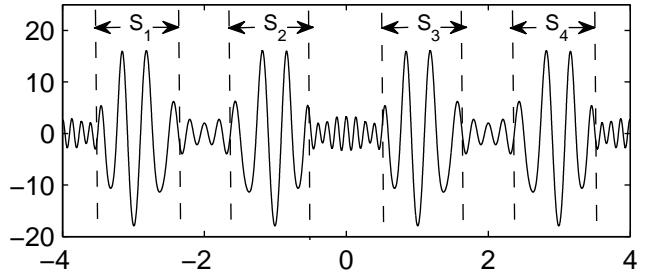

(f)

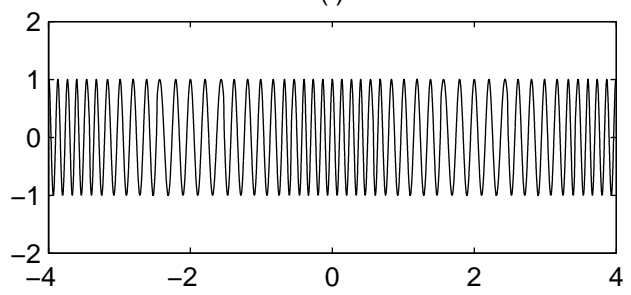

(h)

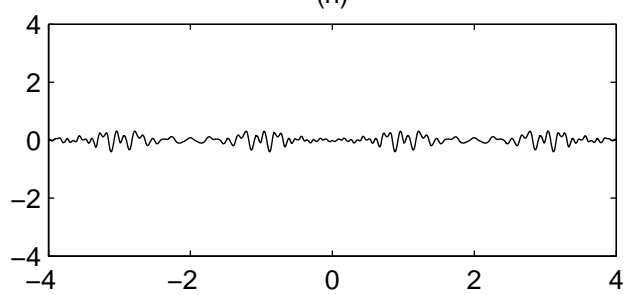

Fig. 4. An example of the mode mixing phenomenon in a two-component AM-FM signal: (a) the input multi-component signal $s(t)=s_{l}(t)+s_{h}(t)$; (b) the first mono-component signal $s_{l}(t)=5(2-1.2 \cos (\pi t)) \cos (6 \pi t+3 \sin (\pi t / 2))$; (c) the second mono-component signal $s_{h}(t)=(2+1.2 \cos (\pi t)) \cos (12 \pi t+6 \sin (\pi t / 2))$; (d) the first extracted IMF of the input signal $s(t)$; (e) the signal $\hat{d}(t)$ used to calculate the masking signal; (f) the normalized masking signal $\hat{m}(t)$; (g) and (h) the first subcomponent extracted by Algorithm 2 and the difference from the real subcomponent $s_{h}(t)$ respectively.

To analyze the properties of $g(t)$ and $d(t)$, we divide the IMF $g(t)$ into $g_{\mathbf{S}}(t)$ and $g_{\overline{\mathbf{S}}}(t)$ as follows:

$$
g(t)=g_{\mathbf{S}}(t)+g_{\overline{\mathbf{S}}}(t)=g(t) R_{\mathbf{S}}(t)+g(t) R_{\overline{\mathbf{S}}}(t),
$$

where $R_{\mathbf{S}}(t)$ and $R_{\overline{\mathbf{S}}}(t)$ are the indicator functions of $\mathbf{S}$ and $\overline{\mathbf{S}}$ respectively. The indicator function is defined as $R_{A}(t)=1$ for $t \in A$ and $R_{A}(t)=0$ for $t \notin A$. Because $g(t)$ only contains information about $s_{h}(t)$ when $t \in \overline{\mathbf{S}}$, we assume that $\tilde{a}_{l}(t)=0$ and conclude that $g_{\overline{\mathbf{S}}}(t)=\tilde{a}_{h}(t) \cos \left(\tilde{\phi}_{h}(t)\right)$. Moreover, when 
the mode-mixing phenomenon occurs, the extrema on segments in $\mathbf{S}$ are contributed primarily by the low frequency FM component $s_{l}(t)$. In this case, $s_{h}(t)$ is almost immersed in $s_{l}(t)$; therefore, $a_{l}(t) \gg a_{h}(t)$ for $t \in \mathbf{S}$. If the condition $\left(\tilde{a}_{l}(t) / \tilde{a}_{h}(t)\right)\left(\tilde{\phi}_{l}^{\prime}(t) / \tilde{\phi}_{h}^{\prime}(t)\right)^{2}>1$ is satisfied, we can assume that the positions of the extrema in $g_{\mathbf{S}}(t)$ are very close to the locations of the extrema in $\cos \left(\tilde{\phi}_{l}(t)\right)$. The condition is derived by

$$
\begin{aligned}
g_{\mathbf{S}}(t) & =\tilde{a}_{l}(t) \cos \left(\tilde{\phi}_{l}(t)\right)+\tilde{a}_{h}(t) \cos \left(\tilde{\phi}_{h}(t)\right) \\
& =\tilde{a}_{l}(t) \cos \left(\tilde{\phi}_{l}^{\prime}(t) t+\beta_{l}\right)+\tilde{a}_{h}(t) \cos \left(\tilde{\phi}_{h}^{\prime}(t) t+\beta_{h}\right),
\end{aligned}
$$

where $\beta_{l}$ and $\beta_{h}$ are the phase values at $t=0$. If we assume that $\tilde{a}_{l}(t), \tilde{a}_{h}(t), \tilde{\phi}_{l}^{\prime}(t)$ and $\tilde{\phi}_{h}^{\prime}(t)$ are constants in a sufficiently small neighborhood of $t$, we can also assume that, in the neighborhood of $t$, $g(t)$ can be approximated as the sum of two sine waves. In this case, we can use the analysis results in [12] to derive that the locations of the extrema points of $g_{\mathbf{S}}(t)$ are close to those of $\tilde{a}_{l}(t) \cos \left(\tilde{\phi}_{l}(t)\right)$, and the distance between an extremum of $g_{\mathbf{S}}(t)$ and the corresponding extremum of $\tilde{a}_{l}(t) \cos \left(\tilde{\phi}_{l}(t)\right)$ is less than $1 /(2 \pi f(t)) \arcsin (1 / a(t) f(t))$ with $a(t)=\tilde{a}_{l}(t) / \tilde{a}_{h}(t)$ and $f(t)=\tilde{\phi}_{l}^{\prime}(t) / \tilde{\phi}_{h}^{\prime}(t)$.

Let $E_{p}\{g(t)\}$ denote the extrema points of the signal $g(t)$. Then, we have

$$
\begin{aligned}
E_{p}\{g(t)\} & =E_{p}\left\{g_{\mathbf{S}}(t)\right\}+E_{p}\left\{g_{\overline{\mathbf{S}}}(t)\right\} \\
& \approx E_{p}\left\{\tilde{a}_{l}(t) \cos \left(\tilde{\phi}_{l}(t)\right) R_{\mathbf{S}}(t)\right\}+E_{p}\left\{\tilde{a}_{h}(t) \cos \left(\tilde{\phi}_{h}(t)\right) R_{\overline{\mathbf{S}}}(t)\right\} .
\end{aligned}
$$

Equation (22) is derived because the segments in $\mathbf{S}$ and $\overline{\mathbf{S}}$ form a partition in the signal domain and the end points of segments do not contain the extrema points of $g(t)$. From $E_{p}\{g(t)\}$, we can approximate $g(t)$ by an AM-FM signal $\hat{g}(t)=\hat{a}(t) \cos (\hat{\phi}(t))^{1}$, where $\hat{a}(t)$ and $\hat{\phi}(t)$ are the estimated AM and FM components respectively. The difference between $g(t)$ and $\hat{g}(t)$ is

$$
\begin{aligned}
d(t) & =g(t)-\hat{g}(t) \\
& =\left(\sum_{i=l, h} \tilde{a}_{i}(t) \cos \left(\tilde{\phi}_{i}(t)\right)-\hat{a}(t) \cos (\hat{\phi}(t))\right) R_{\mathbf{S}}(t)+\left(\tilde{a}_{h}(t) \cos \left(\tilde{\phi}_{h}(t)\right)-\hat{a}(t) \cos (\hat{\phi}(t))\right) R_{\overline{\mathbf{S}}}(t) \\
& =d_{\mathbf{S}}(t)+d_{\overline{\mathbf{S}}}(t) .
\end{aligned}
$$

Next, we discuss the two components in (24).

Case 1. $t \in \overline{\mathbf{S}}$ :

\footnotetext{
${ }^{1}$ The steps for computing $\hat{g}(t)$ are given in the remarks of Algorithm 2
} 
As mentioned earlier, we have $\tilde{a}_{l}(t)=0$ and $E_{p}\left\{g_{\overline{\mathbf{S}}}(t)\right\}=E_{p}\left\{\tilde{a}_{h}(t) \cos \left(\tilde{\phi}_{h}(t)\right)\right\}$. The AM-FM signal $\hat{h}(t)=\hat{a}(t) \cos (\hat{\phi}(t))$, estimated from the extrema of $g_{\overline{\mathbf{S}}}(t)$ by EMD, will have $\hat{a}(t) \approx \tilde{a}_{h}(t)$ and $\hat{\phi}(t) \approx \tilde{\phi}_{h}(t)$. Thus, the absolute difference in $\overline{\mathbf{S}}$ is bounded by a small value $\epsilon$; i.e., $\left|d_{\overline{\mathbf{S}}}(t)\right|<\epsilon$.

Case 2. $t \in \mathbf{S}$ :

Although $g_{\mathbf{S}}(t)=\tilde{a}_{l}(t) \cos \left(\tilde{\phi}_{l}(t)\right)+\tilde{a}_{h}(t) \cos \left(\tilde{\phi}_{h}(t)\right)$, the positions of the extrema of $g_{\mathbf{S}}(t)$ are close to those of the low frequency FM component $\cos \left(\tilde{\phi}_{l}(t)\right)$. Thus, we can assume that $\hat{\phi}(t) \approx \tilde{\phi}_{l}(t)$. On the other hand, the amplitude of the extrema of $g_{\mathbf{S}}(t)$ cannot be approximated as $\tilde{a}_{l}(t)$ because $g_{\mathbf{S}}(t)$ also contains the other component $\tilde{a}_{h}(t) \cos \left(\tilde{\phi}_{h}(t)\right)$. We assume that $\hat{a}(t)=\tilde{a}_{l}(t)+\Delta a(t)$. Since both $\hat{a}(t)$ and $\tilde{a}_{l}(t)$ are smoothly varying functions, $\Delta a(t)$ is also a smoothly varying function. As shown in (25), the difference between $g(t)$ and $\hat{g}(t)$ is

$$
\begin{aligned}
d_{\mathbf{S}}(t) & =\sum_{i=l, h} \tilde{a}_{i}(t) \cos \left(\tilde{\phi}_{i}(t)\right)-\hat{a}(t) \cos (\hat{\phi}(t)) \\
& =\left(\tilde{a}_{l}(t)\left(\cos \left(\tilde{\phi}_{l}(t)\right)-\cos (\hat{\phi}(t))\right)-\Delta a(t) \cos (\hat{\phi}(t))\right)+\tilde{a}_{h}(t) \cos \left(\tilde{\phi}_{h}(t)\right) \\
& =-\left(2 \tilde{a}_{l}(t) \sin \left(\left(\tilde{\phi}_{l}(t)-\hat{\phi}(t)\right) / 2\right) \sin \left(\left(\tilde{\phi}_{l}(t)+\hat{\phi}(t)\right) / 2\right)+\Delta a(t) \cos (\hat{\phi}(t))\right)+\tilde{a}_{h}(t) \cos \left(\tilde{\phi}_{h}(t)\right) \\
& \approx-\left(\tilde{a}_{l}(t)\left(\tilde{\phi}_{l}(t)-\hat{\phi}(t)\right) \sin (\hat{\phi}(t))+\Delta a(t) \cos (\hat{\phi}(t))\right)+\tilde{a}_{h}(t) \cos \left(\tilde{\phi}_{h}(t)\right) \\
& =\tilde{a}_{h}(t) \cos \left(\tilde{\phi}_{h}(t)\right)-\tilde{a}_{l}(t) \sqrt{\left(\tilde{\phi}_{l}(t)-\hat{\phi}(t)\right)^{2}+\left(\frac{\Delta a(t)}{\tilde{a}_{l}(t)}\right)^{2}} \sin (\hat{\phi}(t)+\alpha(t))
\end{aligned}
$$

where $\alpha(t)=\arctan \left(\frac{\Delta a(t)}{\tilde{a}_{l}(t)\left(\tilde{\phi}_{l}(t)-\hat{\phi}(t)\right)}\right)$. To calculate (27) and (28), we use the fact that $\tilde{\phi}_{l}(t) \approx \hat{\phi}(t)$ and the approximations $\left(\tilde{\phi}_{l}(t)+\hat{\phi}(t)\right) / 2 \approx \hat{\phi}(t)$ and $\sin \left(\tilde{\phi}_{l}(t)-\hat{\phi}(t)\right) \approx \tilde{\phi}_{l}(t)-\hat{\phi}(t)$.

In Appendix $\mathrm{B}$, we show that if $\tilde{\phi}_{l}(t)-\hat{\phi}(t) \approx 0$, we can estimate $\tilde{\phi}_{l}(t)$ accurately when $t \in S$, and $\left(|\Delta a(t)| / \tilde{a}_{h}(t)\right)\left(\tilde{\phi}_{l}^{\prime}(t) / \tilde{\phi}_{h}^{\prime}(t)\right)<1$. Then, we have

$$
E_{p}\left\{d_{\mathbf{S}}(t)\right\} \approx E_{p}\left\{\tilde{a}_{h}(t) \cos \left(\tilde{\phi}_{h}(t)\right)\right\}
$$

Equation (30) indicates that the extrema of the hidden high frequency component $s_{h}(t)$ can be retrieved from $d_{\mathbf{S}}(t)$. On the other hand, $d_{\overline{\mathbf{S}}}(t)$ is upper bounded by a small $\epsilon$; thus, we can apply a threshold or some morphological operators on $d(t)$ such that the values of $d$ on segments $\overline{\mathbf{S}}$ become zero. The resultant signal $\hat{d}(t)$ is $d_{\mathbf{S}}(t)$ for $t \in \mathbf{S}$, and it has a zero value for $t \in \overline{\mathbf{S}}$. Figure 4(e) plots the derived $\hat{d}(t)$ for the example in Figure 4(d). Note that in segments $S_{1}$ to $S_{4}, d(t)$ contains the high frequency component of Figure 4(c) and the rest are zero. 


\section{B. Constructing a masking signal}

To construct a masking signal, we form the following signal $y(t)=g(t)+\lambda \hat{d}(t)$, where $\lambda>0$. Because $\hat{d}_{\overline{\mathbf{S}}}(t)=0$, we have

$$
y(t)=g_{\mathbf{S}}(t)+\lambda \hat{d}_{\mathbf{S}}(t)+g_{\overline{\mathbf{S}}}(t)
$$

Calculating the extrema of $y(t)$, we obtain

$$
\begin{aligned}
E_{p}\{y(t)\} & =E_{p}\left\{g_{\mathbf{S}}(t)+\lambda \hat{d}_{\mathbf{S}}(t)+g_{\overline{\mathbf{S}}}(t)\right\} \\
& =E_{p}\left\{g_{\mathbf{S}}(t)+\lambda \hat{d}_{\mathbf{S}}(t)\right\}+E_{p}\left\{g_{\overline{\mathbf{S}}}(t)\right\}+E_{y}(t),
\end{aligned}
$$

where $E_{y}(t)$ is the extrema of $y(t)$ at the intersection points of two segments on $y(t)$; one is in $\mathbf{S}$ and the other is in $\overline{\mathbf{S}}$. If we substitute (30) into (32) and use the fact that

$$
g_{\mathbf{S}}(t)=\left(\tilde{a}_{l}(t) \cos \left(\tilde{\phi}_{l}(t)\right)+\tilde{a}_{h}(t) \cos \left(\tilde{\phi}_{h}(t)\right)\right) R_{\mathbf{S}}(t)
$$

and

$$
g_{\overline{\mathbf{S}}}(t)=\left(\tilde{a}_{h}(t) \cos \left(\tilde{\phi}_{h}(t)\right)\right) R_{\overline{\mathbf{S}}}(t)
$$

we have

$E_{p}\{y(t)\}=E_{p}\left\{\left(\tilde{a}_{l}(t) \cos \left(\tilde{\phi}_{l}(t)\right)+(1+\lambda) \tilde{a}_{h}(t) \cos \left(\tilde{\phi}_{h}(t)\right)\right) R_{\mathbf{S}}\right\}+E_{p}\left\{\tilde{a}_{h}(t) \cos \left(\tilde{\phi}_{h}(t)\right) R_{\overline{\mathbf{S}}}(t)\right\}+E_{y}(t)$.

We can select a $\lambda$ value with $(1+\lambda) \tilde{a}_{h}(t)>\tilde{a}_{l}(t)$ so that, for $t \in \mathbf{S}$, we have

$$
E_{p}\left\{\left(\tilde{a}_{l}(t) \cos \left(\tilde{\phi}_{l}(t)\right)+(1+\lambda) \tilde{a}_{h}(t) \cos \left(\tilde{\phi}_{h}(t)\right)\right) R_{\mathbf{S}}\right\} \approx E_{p}\left\{\left((1+\lambda) \tilde{a}_{h}(t) \cos \left(\tilde{\phi}_{h}(t)\right)\right) R_{\mathbf{S}}\right\} .
$$

Substituting (36) into (35), we can derive that

$$
E_{p}\{y(t)\} \approx E_{p}\left\{\left((1+\lambda) \tilde{a}_{h}(t) \cos \left(\tilde{\phi}_{h}(t)\right)\right) R_{\mathbf{S}}(t)\right\}+E_{p}\left\{\tilde{a}_{h}(t) \cos \left(\tilde{\phi}_{h}(t)\right) R_{\overline{\mathbf{S}}}(t)\right\}+E_{y}(t) .
$$

Usually, the number of extrema in $E_{y}(t)$ is much smaller than in $\tilde{a}_{h}(t) \cos \left(\tilde{\phi}_{h}(t)\right)$; thus, we can omit the term $E_{y}(t)$ in (37) and obtain

$$
E_{p}\{y(t)\} \approx E_{p}\left\{\left((1+\lambda) \tilde{a}_{h}(t) \cos \left(\tilde{\phi}_{h}(t)\right)\right) R_{\mathbf{S}}(t)\right\}+E_{p}\left\{\tilde{a}_{h}(t) \cos \left(\tilde{\phi}_{h}(t)\right) R_{\overline{\mathbf{S}}}(t)\right\} .
$$

Because $\tilde{a}_{h}(t)$ is a slowly varying function, the positions of the extrema of $y(t)$ are close to those of $\cos \left(\tilde{\phi}_{h}(t)\right)$. Thus, the IMF $\tilde{m}(t)$ of $y(t)$ should maintain a similar frequency function to $\tilde{\phi}_{h}(t)$. Through the AM-FM demodulation of $\tilde{m}(t)$, a normalized $\hat{m}(t)$ can be obtained by dividing $\tilde{m}(t)$ by the derived envelope. The $\hat{m}(t)$ is our masking signal, which is an FM signal of the form $\cos \left(\hat{\phi}_{h}(t)\right)$ with $\hat{\phi}_{h}^{\prime}(t) \approx$ $\tilde{\phi}_{h}^{\prime}(t) \approx \phi_{h}^{\prime}(t)$. The masking signal of Figure 4(d) is shown in Figure 4(f). 


\section{Recovering the desired IMF, and evaluations and comparisons}

To recover the desired hidden subcomponent signal $s_{h}(t)$ when $t \in \mathbf{S}$, we adopt Deering and Kaiser's method by using $\hat{m}(t)$ as the masking signal. Our method is summarized in Algorithm 2. Steps 3 to 7 of the algorithm estimate our masking signal. The last three steps are from Deering and Kaiser's method, which we use to recover the desired IMF. Figures $4(\mathrm{~g})$ and $(\mathrm{h})$ depict the desired IMF recovered by our method and the difference between the recovered IMF and the ground truth IMF in Figure 4(c).

The difference between Deering and Kaiser's method and our method is the way the masking signal is estimated. Figure 5 compares the results of applying the methods to the mode-mixing problem of a twocomponent FM signal. The IMF extracted by the EMD method is shown in Figure 5(d). It is obviously affected by the mode-mixing problem. The masking signals of Deering and Kaiser's method and our method are shown in Figures $5(\mathrm{~h})$ and 5(g) respectively. Deering and Kaiser use a pure sine wave as the masking signal. The frequency is estimated from the weighted mean of the extracted IMF's frequency acquired by the Hilbert transform. The IMF extracted by their approach, shown in Figure 5(f), is not the desired IMF of the input signal. In contrast, our masking signal is an FM signal that solves the problem. Our recovered IMF is shown in Figure 5(e), which corresponds to the high frequency component in Figure 5(b).

Finally, we evaluate the proposed approach on the mode mixing phenomenon in a simulated signal containing more than two AM-FM signals and a real life signal. The multi-component AM-FM signal $s(t)$ and its three subcomponents, $s_{1}(t), s_{2}(t)$, and $s_{3}(t)$, are shown in Figures 6(a),(b),(c), and (d) respectively. Although $s_{1}(t)$ has the highest frequency, $s_{2}(t)$ has the second highest, and $s_{3}(t)$ has the lowest frequency, the amplitude of the lowest frequency component $s_{3}(t)$ is larger than those of the other two components. Consequently, the signal $s(t)$ appears to be of low frequency. The first desired IMF is $s_{1}(t)$ and the second desired IMF is $s_{2}(t)$; however, the first IMF extracted by EMD is not $s_{1}(t)$, as shown in Figure 7(a). The IMF is obviously affected by the mode-mixing problem, as shown in segments $S_{1}$ to $S_{5}$. In segments $S_{1}, S_{2}, S_{4}$, and $S_{5}$, we can only observe the low frequency information about $s_{3}(t)$; while segment $S_{3}$ contains the information about $s_{1}(t)$ as well as the information about $s_{2}(t)$. We resolve the mode-mixing problem in the IMF by applying our method with the estimated masking signal, as shown in Figure 7(b). The resulting IMF, shown in Figure 7(c), is the desired high frequency IMF $\hat{s}_{1}(t)$. Then, EMD is applied on the signal $s(t)-\hat{s}_{1}(t)$ and the IMF is extracted, as shown in Figure 7(d). This IMF is also affected by the mode-mixing problem. As shown in segments $S_{1}$ and $S_{2}$ in Figure $7(\mathrm{~d})$, the high frequency information of $s_{2}(t)$ is hidden in the low frequency component $s_{3}(t)$. We then 
(a)

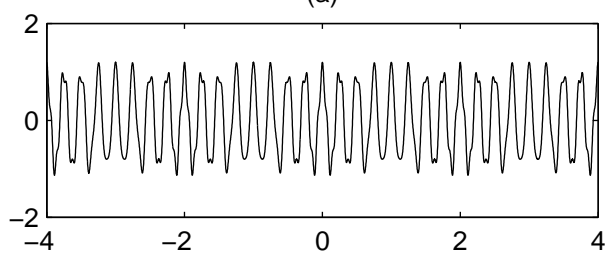

(c)

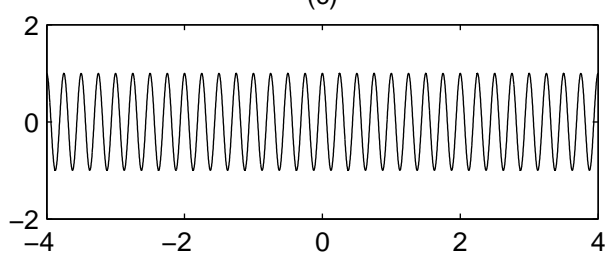

(e)

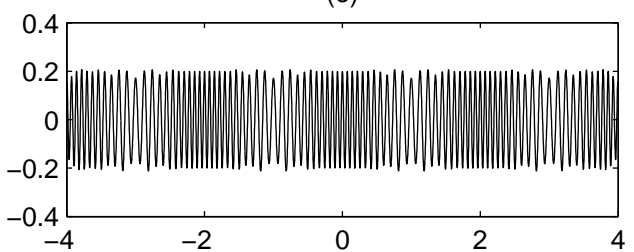

(g)

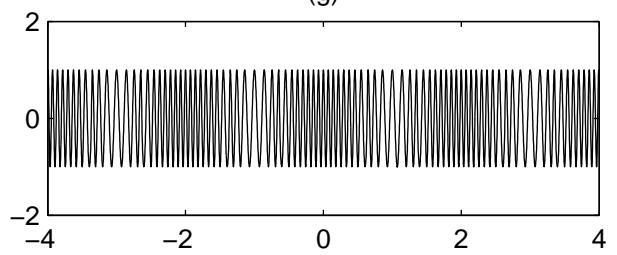

(b)

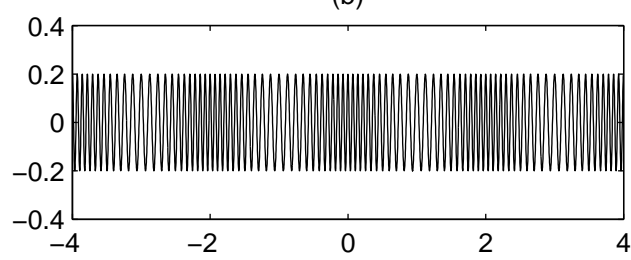

(d)

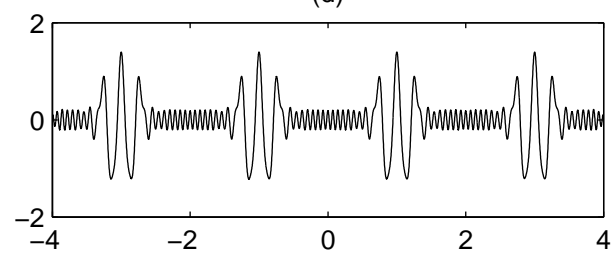

(f)

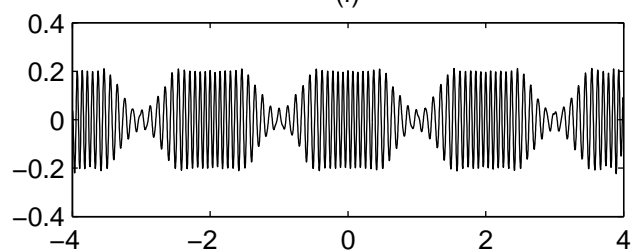

(h)

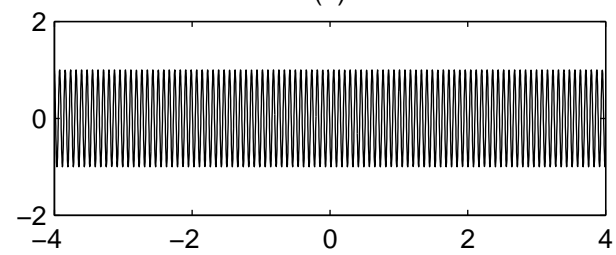

Fig. 5. Comparison of the results derived by our method and Deering and Kaiser's method. (a) The input two component FM signal: $s_{1}(t)$ and $s_{2}(t)$ in (b) and (c) respectively; (b) the signal $s_{1}(t)$ is $0.2 \cos (22 \pi t+6 \sin (\pi t))$; (c) the signal $s_{2}(t)$ is $\cos (8 \pi t)$; (d) the first IMF extracted by EMD from the input signal in (a). This IMF has a mode-mixing problem; (e) and (f) the extracted signals derived by our algorithm and Deering and Kaiser's method respectively; (g) and (h) the estimated masking signals of our algorithm and Deering and Kaiser's method respectively. Since Deering and Kaiser's method can only construct a pure sine wave mask, it cannot separate the signal well in the segments where the signal's frequencies are not close to that of the mask. Thus, in those segments, the desired component cannot be extracted by the masking signal. A close inspection reveals that our masking signal's frequency is not exactly the same as the frequency of $s_{1}(t)$, but it is very close. Hence, our masking signal can extract the desired subcomponent.

apply our method to the IMF with the masking signal derived in Figure 7(e). The resulting IMF is shown in Figure 7(f), which is the desired IMF $\hat{s}_{2}(t)$. The residual signal is $\hat{s}_{3}(t)$, shown in Figure $7(\mathrm{~g})$. This example demonstrates that the proposed method can successfully resolve the mode-mixing problem in more than two AM-FM signals. 
(a)

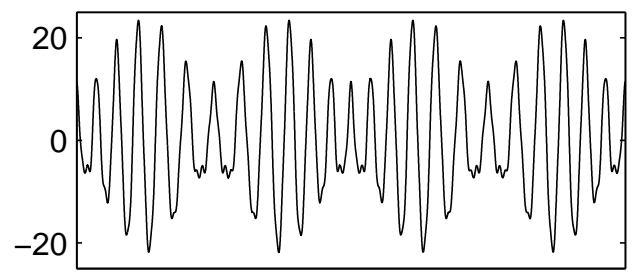

(c)

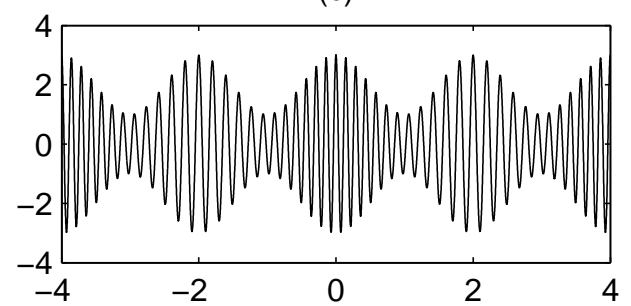

(b)

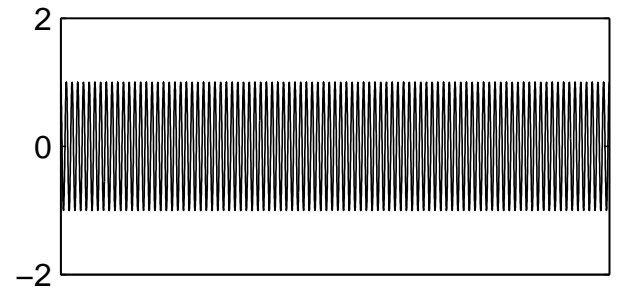

(d)

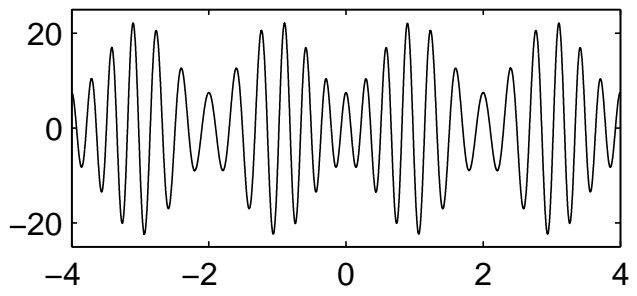

Fig. 6. (a) A multicomponent AM-FM signal is comprised of three subcomponents: $s_{1}(t), s_{2}(t)$, and $s_{3}(t)$ in (b), (c), and (d) respectively; (b) the signal $s_{1}(t)$ is $\cos (18 \pi t)$; (c) the signal $s_{2}(t)$ is $(2+\cos (\pi t)) \cos \left(12 \pi t+4 \sin \left(\frac{\pi}{2} t\right)\right)$; (d) the signal $s_{3}(t)$ is $7.5(2-\cos (\pi t)) \cos \left(6 \pi t+2 \sin \left(\frac{\pi}{2} t\right)\right) ; s_{1}(t)$ has the highest frequency, $s_{2}(t)$ has the second highest, and $s_{3}(t)$ has the lowest frequency.

Our real-life signal is the airline passenger data from [26]. The data has 144 points corresponding to the 144 months in a 12-year period. The input data and its spectrum are shown in Figures 8 (a) and 8(b) respectively. Since a real-life signal always contains noise, we use the ensemble EMD algorithm (EEMD), which is more robust than EMD, to decompose a noisy signal. Figures 8(c),(e), (g), and (i) correspond to the first, second, third, and fourth IMFs of the EEMD algorithm; Figure 8(k) is the residual. We observe that the first and second IMFs have a mixture of extrema with different rates. Thus, we apply the proposed method to the IMF in Figure 8(c) and derive our first IMF, as shown in Figure 8(d). After subtracting that IMF from the input signal, we apply the EMD algorithm on the resulting signal and obtain another IMF. We then apply our method to that IMF and obtain our second IMF, which is shown in Figure 8(f). Our third and fourth IMFs and our residual signals are shown in Figure 8(h), (j) and (l) respectively. The spectrograms of the Gabor transform on the IMFs in Figures 8(c) and (d) are shown in Figures 9(a) and 9(b) respectively. Because the first IMF derived by EEMD is not an AM-FM signal, shown in Figure 8(c), our analysis of the AM-FM signals cannot be applied to the signal correctly. However, if we compare the spectrograms of Figures 9(a) and 9(b), the pattern of our IMF, as shown in Figure 9(b), 
(a)

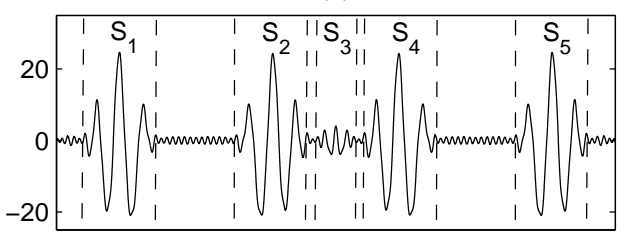

(b)

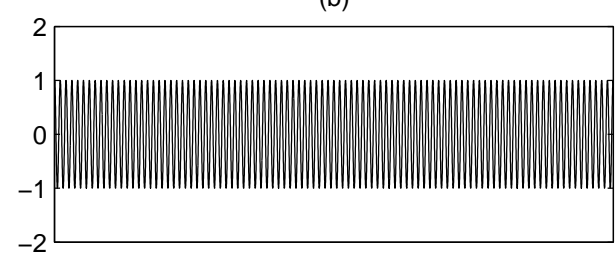

(c)

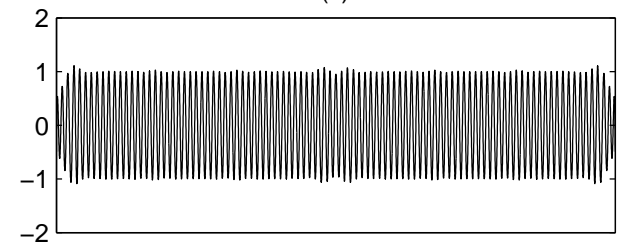

(g)

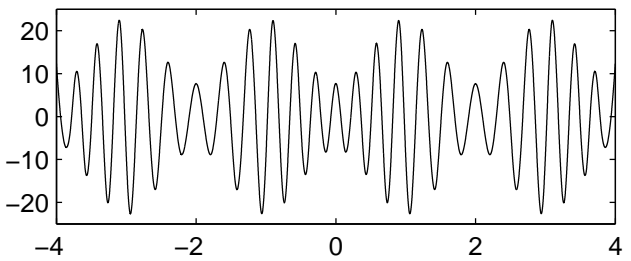

(d)

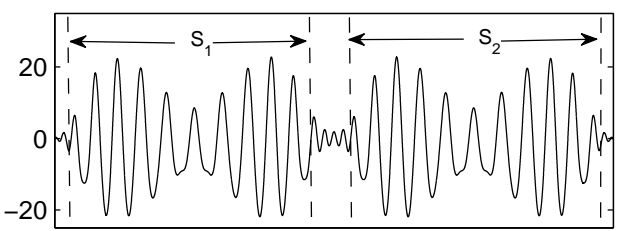

(e)

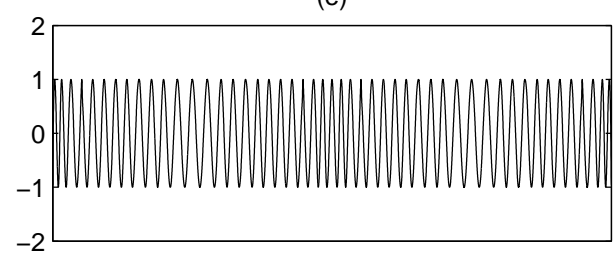

(f)

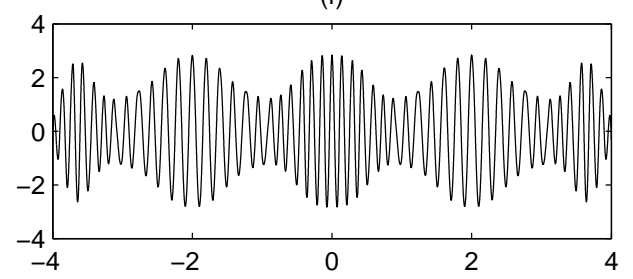

Fig. 7. (a) The first IMF extracted by EMD from the signal in Figure 6(a). Although the high frequency information other than segments $S_{1}$ to $S_{5}$ is correctly extracted, the information is hidden in the segments; (b) our FM masking signal, derived from (a); (c) our first extracted IMF, which is the desired IMF $\hat{s}_{1}(t)$; (d): the IMF of $s(t)-\hat{s}_{1}(t)$, where $s(t)$ and $\hat{s}_{1}(t)$ are shown in Figures 6(a) and 7(c) respectively. The mode-mixing phenomenon appears in segments $S_{1}$ and $S_{2}$; (e) our FM masking signal, derived from (d); (f) our extracted IMF which is $\hat{s}_{2}(t)$, the desired IMF of $s(t)-\hat{s}_{1}(t)$; and (g) the residual signal, which is $\hat{s}_{3}(t)$.

is more regular than that of the EEMD algorithm shown Figure 9(a). The spectrograms of the Gabor transform for the second IMFs in Figures 8(e) and 8(f) are shown in Figures 9(c) and 9(d) respectively. As shown in Figure 9(c), in the rectangle enclosed by the dashed lines, the IMF of the EEMD has more than two distinctive frequencies in the interval between 70 and 120; while our IMF, shown in Figure 9(d) only contains one dominant frequency. 
(a)

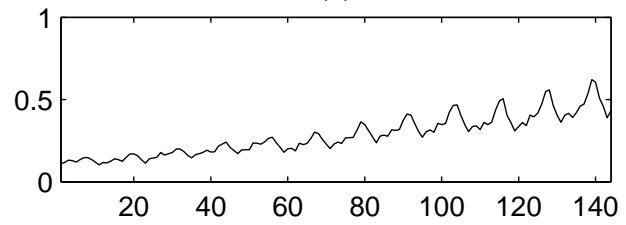

(c)

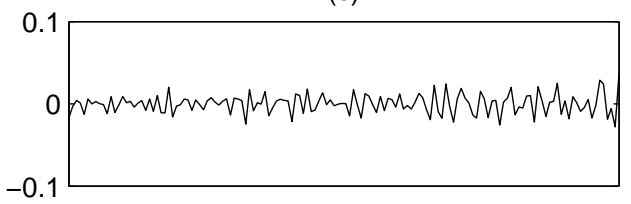

(e)

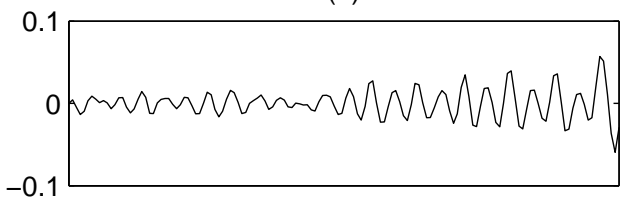

(g)

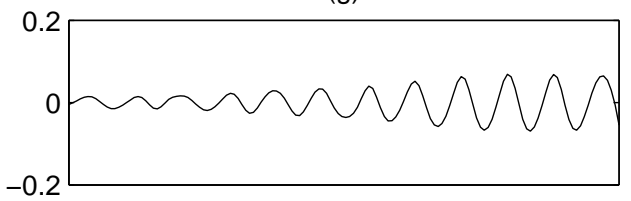

(i)

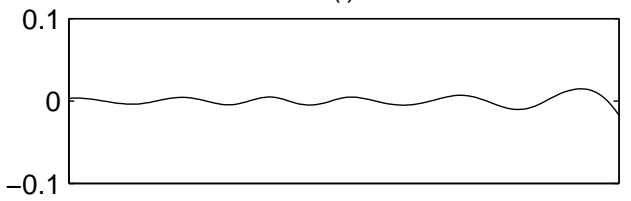

(k)

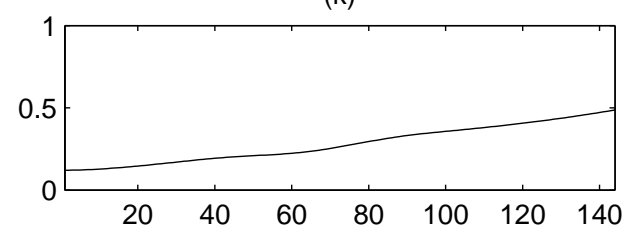

(b)

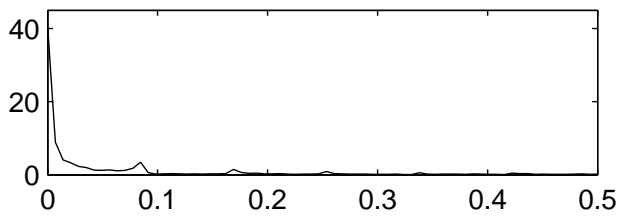

(d)

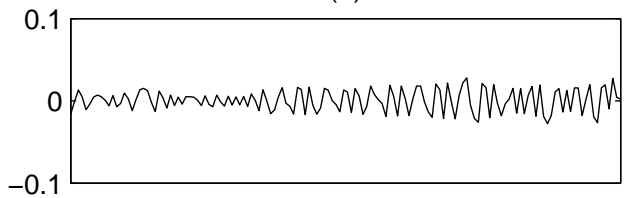

(f)

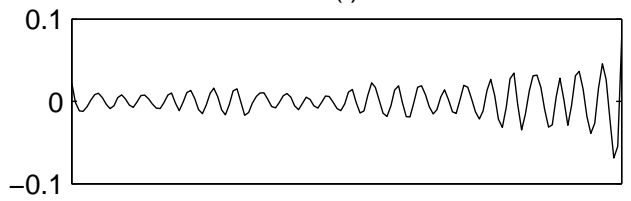

(h)

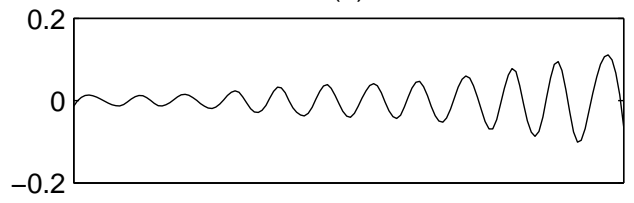

(j)

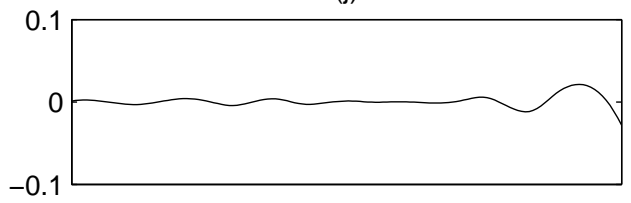

(I)

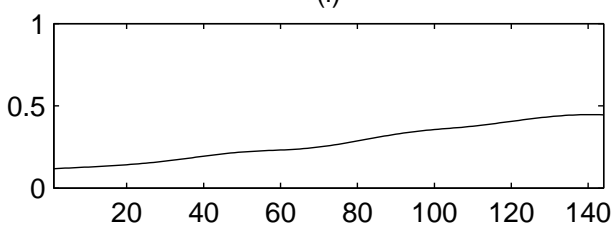

Fig. 8. Analysis of the airline passenger data: (a) the input signal; (b) the input signal's Fourier spectrum. (c),(e),(g),and (i) are the first, second, third, and fourth IMFs derived by applying the EEMD algorithm to the input signal. The value of the parameter $\sigma$ in EEMD is 2.5 and the ensemble number is 1000 , which means that we add 1000 realizations of Gaussian noise sampled with a standard deviation $\sigma$ to the input signal and then perform EMD on the resultant signal. (d),(f),(h), and (j) are the first, second, third, and fourth IMFs derived by applying our method on the first two IMFs of the EMD method.

\section{CONCLUding REMARKS}

We propose a new necessary condition on the IMF of EMD. In addition, we introduce an approximation algorithm to estimate the envelope of an AM-FM signal. We demonstrate that the envelope does not 
(a)

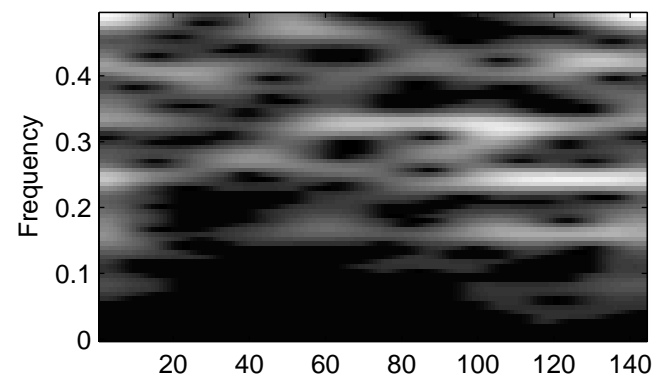

(c)

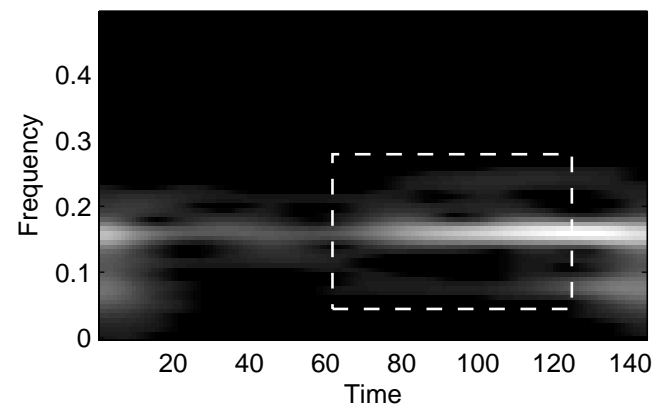

(b)

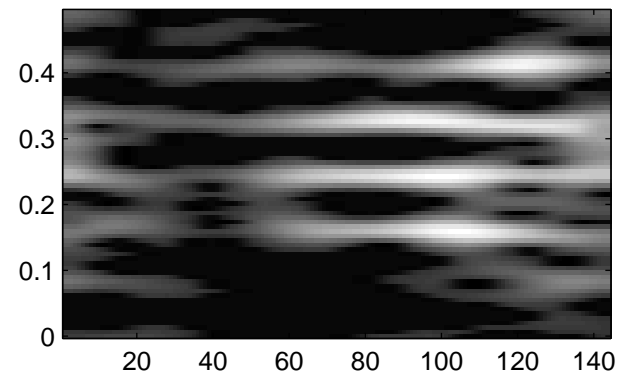

(d)

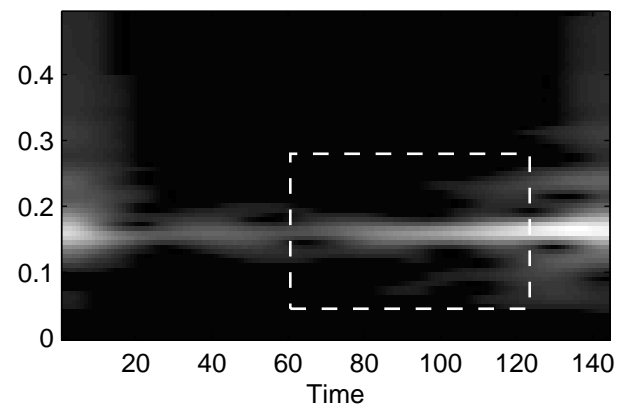

Fig. 9. The spectrograms of the Gabor transform for IMFs; (a) the spectrogram of the first IMF derived by EEMD; (b) the spectrogram of the first IMF, derived by our algorithm. The first IMF derived by EEMD is not an AM-FM signal; (c) and (d) the spectrograms of the IMFs derived by the EEMD algorithm and our algorithm respectively. The frequency axis is normalized between 0 and 0.5. Comparing the regions enclosed by the dashed lines, the IMF derived by EEMD is affected by the mode-mixing phenomenon because (c) has two distinct frequencies in the corresponding interval from 70 to 120 .

necessarily pass through the extrema of an IMF. Furthermore, based on the AM-FM signal model, we generalize Deering and Kaiser's approach to resolve the mode-mixing problem in the IMFs of AM-FM signals. We evaluate our approach via simulations and an experiment on real-life signals. Our research is a step towards gaining a clearer understanding of the envelope of the EMD method. Providing a clear mathematical definition of the envelope is an important issue that warrants further study. Moreover, in many real-life signals, the IMF of the EMD method cannot be modeled as a multicomponent AM-FM signal. To extend our mode-mixing method to such signals is another issue that merits further study.

\section{ACKNOWLEDGMENTS}

The authors wish to thank the reviewers and the associate editor for their insightful comments, which helped us improve the quality of the paper significantly. 


\section{APPENDIX}

\section{A. The convergence of Algorithm 1}

We use the second order Taylor expansion of $s\left(t_{i}^{(k+1)}\right)$ in (11) to obtain

$$
\psi^{(k+1)}\left(t_{i}^{(k+1)}\right)=s\left(t_{i}^{(k)}+\Delta t_{i}^{(k)}\right)=s\left(t_{i}^{(k)}\right)+s^{\prime}\left(t_{i}^{(k)}\right) \Delta t_{i}^{(k)}+0.5 s^{\prime \prime}\left(t_{i}^{(k)}\right) \Delta t_{i}^{(k)^{2}}+o\left(\Delta t_{i}^{(k)^{2}}\right) .
$$

Substituting $\psi^{(k+1)}\left(t_{i}^{(k+1)}\right)$ in Equation (39) and $\psi^{(k+1)}\left(t_{i}^{(k)}\right)$ in (13) into equation (12), we have

$$
\psi^{(k+1)^{\prime}}\left(t_{i}^{(k+1)}\right)=\psi^{(k)^{\prime}}\left(t_{i}^{(k)}\right)+0.5 \psi^{(k)^{\prime \prime}}\left(t_{i}^{(k)}\right) \Delta t_{i}^{(k)}+o\left(\Delta t_{i}^{(k)}\right) .
$$

Then, from equations (40) and (10), we can obtain the offset $\Delta t_{i}^{(k+1)}$ :

$$
\Delta t_{i}^{(k+1)}=\frac{s^{\prime}\left(t_{i}^{(k+1)}\right)-\left(\psi^{(k)^{\prime}}\left(t_{i}^{(k)}\right)+0.5 \psi^{(k)^{\prime \prime}}\left(t_{i}^{(k)}\right) \Delta t_{i}^{(k)}+o\left(\Delta t_{i}^{(k)}\right)\right)}{\psi^{(k+1)^{\prime \prime}}\left(t_{i}^{(k+1)}\right)-s^{\prime \prime}\left(t_{i}^{(k+1)}\right)} .
$$

If we apply the first order Taylor expansion on $s^{\prime}\left(t^{(k+1)}\right)$ in equation (41), then we have

$$
\Delta t_{i}^{(k+1)}=\frac{s^{\prime}\left(t_{i}^{(k)}\right)-\psi^{(k)^{\prime}}\left(t_{i}^{(k)}\right)+\left(s^{\prime \prime}\left(t_{i}^{(k)}\right)-\psi^{(k)^{\prime \prime}}\left(t_{i}^{(k)}\right)\right) \Delta t_{i}^{(k)}}{\psi^{(k+1)^{\prime \prime}}\left(t_{i}^{(k+1)}\right)-s^{\prime \prime}\left(t_{i}^{(k+1)}\right)}+\frac{0.5 \psi^{(k)^{\prime \prime}}\left(t_{i}^{(k)}\right) \Delta t_{i}^{(k)}+o\left(\Delta t_{i}^{(k)}\right)}{\psi^{(k+1)^{\prime \prime}}\left(t_{i}^{(k+1)}\right)-s^{\prime \prime}\left(t_{i}^{(k+1)}\right)} .
$$

Substituting $\Delta t_{i}^{(k)}$ in equation (10) into equation (42) and ignoring the high order term $o\left(\Delta t_{i}^{(k)}\right)$, we can derive

$$
\Delta t_{i}^{(k+1)} \approx \frac{0.5 \psi^{(k)^{\prime \prime}}\left(t_{i}^{(k)}\right) \Delta t_{i}^{(k)}}{\psi^{(k+1)^{\prime \prime}}\left(t_{i}^{(k+1)}\right)-s^{\prime \prime}\left(t_{i}^{(k+1)}\right)}=\alpha_{i}^{k} \Delta t_{i}^{(k)}
$$

Since the point $t_{i}^{(k)}$ is near the local maximum point of the signal when computing the upper envelope, we can assume that $s^{\prime \prime}\left(t_{i}^{(k+1)}\right)<0$. We can also assume that an envelope varies much more slowly than a signal; therefore, $\left|\psi^{(k)^{\prime \prime}}\left(t_{i}^{(k)}\right)\right| \approx\left|\psi^{(k+1)^{\prime \prime}}\left(t_{i}^{(k+1)}\right)\right|<2 / 3\left|s^{\prime \prime}\left(t_{i}^{(k+1)}\right)\right|$. If the two assumptions are verified, then we have $\left|\alpha_{i}^{k}\right|<1$.

Thus, we conclude that if there is some integer $l$ such that $s^{\prime \prime}\left(t_{i}^{(k+1)}\right)<0$ and $\left|\psi^{(k)^{\prime \prime}}\left(t_{i}^{(k)}\right)\right| \approx$ $\left|\psi^{(k+1)^{\prime \prime}}\left(t_{i}^{(k+1)}\right)\right|<2 / 3\left|s^{\prime \prime}\left(t_{i}^{(k+1)}\right)\right|$ for all $k \geq l$ and for all $i$, then Algorithm 1 converges.

\section{B. The sufficient condition to derive Equation (30)}

Let us consider Equation (29) where

$$
d_{\mathbf{S}}(t)=g_{\mathbf{S}}(t)-\hat{g}_{\mathbf{S}}(t)=\tilde{a}_{h}(t) \cos \left(\tilde{\phi}_{h}(t)\right)-\sqrt{\left(\tilde{\phi}_{l}(t)-\hat{\phi}(t)\right)^{2} \tilde{a}_{l}^{2}(t)+\Delta a^{2}(t)} \sin (\hat{\phi}(t)+\alpha(t)),
$$


where $\hat{g}_{\mathbf{S}}(t)=\hat{a}(t) \cos (\hat{\phi}(t)), \Delta a(t)=\hat{a}(t)-\tilde{a}_{l}(t)$, and $\alpha(t)=\arctan \left(\frac{\Delta a(t)}{\tilde{a}_{l}(t)\left(\tilde{\phi}_{l}(t)-\hat{\phi}(t)\right)}\right)$ for $t \in \mathbf{S}$. Equation (44) can be re-written as

$$
d_{\mathbf{S}}(t)=\tilde{a}_{h}(t) \cos \left(\tilde{\phi}_{h}^{\prime}(t) t+\beta_{h}\right)+\sqrt{\left(\tilde{\phi}_{l}(t)-\hat{\phi}(t)\right)^{2} \tilde{a}_{l}^{2}(t)+\Delta a^{2}(t)} \cos \left((\hat{\phi}(t)+\alpha(t))^{\prime} t+3 \pi / 2+\beta_{l}\right),
$$

where $\beta_{l}$ and $\beta_{h}$ are the phase values at $t=0$. If we assume that there is a neighborhood of $t$, where $\tilde{a}(t)$, $\tilde{\phi}_{h}^{\prime}(t), \sqrt{\left(\tilde{\phi}_{l}(t)-\hat{\phi}(t)\right)^{2} \tilde{a}_{l}^{2}(t)+\Delta a^{2}(t)}$, and $(\hat{\phi}(t)+\alpha(t))^{\prime}$ are constants, then we can use the analysis results in [12], as mentioned in Section IV-A, to approximate $d_{\mathbf{S}}(t)$ as the sum of two sine waves in the neighborhood of $t$. Let

$$
a(t)=\sqrt{\left(\tilde{\phi}_{l}(t)-\hat{\phi}(t)\right)^{2} \tilde{a}_{l}^{2}(t)+\Delta a^{2}(t)} / \tilde{a}_{h}(t)
$$

and

$$
f(t)=(\hat{\phi}(t)+\alpha(t))^{\prime} / \tilde{\phi}_{h}^{\prime}(t)
$$

If we use the following inequality:

$$
\sqrt{\left(\tilde{\phi}_{l}(t)-\hat{\phi}(t)\right)^{2} \tilde{a}_{l}^{2}(t)+\Delta a^{2}(t)}<\left|\tilde{\phi}_{l}(t)-\hat{\phi}(t)\right| \tilde{a}_{l}(t)+|\Delta a(t)|,
$$

and assume that $\left|\tilde{\phi}_{l}(t)-\hat{\phi}(t)\right| \approx 0$, we obtain

$$
a(t)<\left(\left|\tilde{\phi}_{l}(t)-\hat{\phi}(t)\right| \tilde{a}_{l}(t)+|\Delta a(t)|\right) / \tilde{a}_{h}(t) \approx|\Delta a(t)| / \tilde{a}_{h}(t) .
$$

The assumption, $\left|\tilde{\phi}_{l}(t)-\hat{\phi}(t)\right| \approx 0$, can also be used to derive that $\alpha(t)$ in (29) can be approximated as $\pi / 2$; therefore, $\alpha^{\prime}(t)=0$. As discussed in Section IV-A, the extrema detected on $g_{\mathbf{S}}(t)$ are very close to the local maximum points of $\left|\tilde{a}_{l}(t) \cos \left(\tilde{\phi}_{l}(t)\right)\right|$. Thus, we have $(\hat{\phi}(t)+\alpha(t))^{\prime} \approx \tilde{\phi}_{l}^{\prime}(t)<\tilde{\phi}_{h}^{\prime}(t)$, such that

$$
f(t)=\tilde{\phi}_{l}^{\prime}(t) / \tilde{\phi}_{h}^{\prime}(t)<1 \text {. }
$$

Summarizing our discussion, if we have $\left|\tilde{\phi}_{l}(t)-\hat{\phi}(t)\right| \approx 0$ and

$$
a(t) f(t)=\left(|\Delta a(t)| / \tilde{a}_{h}(t)\right)\left(\tilde{\phi}_{l}^{\prime}(t) / \tilde{\phi}_{h}^{\prime}(t)\right)<1
$$

then, according to [12], the extrema of $d_{\mathbf{S}}(t)$ are very close to the extrema of the high frequency component, which is $\tilde{a}_{h}(t) \cos \left(\tilde{\phi}_{h}(t)\right)$. Thus, we can derive Equation (30).

\section{REFERENCES}

[1] N. E. Huang, Z. Shen, S. R. Long, M. L. Wu, H. H. Shih, Q. Zheng, N. C. Yen, C. C. Tung, and H. H. Liu, "The empirical mode decomposition and hilbert spectrum for nonlinear and nonstationary time series analysis," Proc. R. Soc. London A, vol. 454, pp. 903-995, 1998.

[2] Q. Chen, N. E. Huang, S. Riemenschneider, and Y. Xu, "A b-spline approach for empirical mode decompositions," Advances in Computational Mathematics, vol. 24, no. 1, pp. 171-195, 2006. 
[3] G. Rilling, P. Flandrin, and P. Goncalves, "On empirical mode decomposition and its algorithms," in IEEE-EURASIP Workshop on Nonlinear Signal and Image Processing, 2003.

[4] N. E. Huang, Z. Shen, and S. R. Long, "A new view of nonlinear water waves: The hilbert spectrum," Annual Review of Fluid Mechanics, vol. 31, pp. 417-458, 1999.

[5] R. Deering and J. F. Kaiser, "The use of a masking signal to improve empirical mode decomposition," in IEEE International Conference on Acoustics, Speech, and Signal Processing, 2005. Proceedings. (ICASSP '05), vol. 4, 2005, pp. 18-23.

[6] P. F. Pai, "Nonlinear vibration characterization by signal decomposition," Journal of Sound and Vibration, vol. 307, pp. 527-544, 2007.

[7] P. Maragos, J. F. Kaiser, and T. F. Quatieri, “On amplitude and frequency demodulation using energy operators," IEEE Transactions on Signal Processing, vol. 41, no. 4, pp. 1532-1551, 1993.

[8] F. Gianfelici, G. Biagetti, P. Crippa, and C. Turchetti, "Multicomponent am-fm representations: An asymptotically exact approach," IEEE Trans. on Audio, Speech, and Language Processing, vol. 15, no. 3, pp. 823-837, 2007.

[9] M. F. Jabloun, M. V. Leonard, and N. Martin, "A new flexible approach to estimate the ia and if of nonstationary signals of long-time duration," IEEE Transactions on Signal Processing, vol. 55, no. 7, pp. 3633-3644, 2007.

[10] S. Peng and W.-L. Hwang, "Adaptive signal decomposition based on local narrow band signals," IEEE Transactions on Signal Processing, vol. 56, no. 7, pp. 2669-2676, 2008.

[11] — - "Null space pursuit: An operator-based approach to adaptive signal separation," IEEE Transactions on Signal Processing, vol. 58, no. 5, pp. 2475-2483, 2010.

[12] G. Rilling and P. Flandrin, "One or two frequencies? the empirical mode decomposition answers," IEEE Transactions on Signal Processing, vol. 56, no. 1, pp. 85-95, 2008.

[13] Z. Xu, B. Huang, and K. Li, “An alternative envelope approach for empirical mode decomposition," Digital Signal Processing, vol. 20, no. 1, pp. 77-84, 2010.

[14] E. Delechelle, J. Lemoine, and O. Niang, "Empirical mode decomposition: an analytical approach for sifting process," IEEE Signal Processing Letters, vol. 12, no. 11, pp. 764-767, 2005.

[15] Y. Kopsinis and S. McLaughlin, "Investigation and performance enhancement of the empirical mode decomposition method based on a heuristic search optimization approach," IEEE Transactions on Signal Processing, vol. 56, no. 1, pp. 1-13, 2008.

[16] H. Hong, X. Wang, and Z. Tao, "Local integral mean-based sifting for empirical mode decomposition," IEEE Signal Processing Letters, vol. 16, no. 10, pp. 841-844, 2009.

[17] J.-C. Nunes, Y. Bouaoune, E. Delechelle, and O. Niang, "Image analysis by bidimensional empirical mode decomposition," Image and Vision Computing, vol. 21, no. 12, pp. 1019-1026, 2003.

[18] K. Subr, C. Soler, and D. F., "Edge-preserving multiscale image decompostion based on local extrema," ACM Transaction on Graphics (SIGGRAPH Asia 2009), 2009.

[19] Z. Wu, N. E. Huang, and X. Chen, "The multi-dimensional ensemble empirical mode decomposition method," Advances in Adaptive Data Analysis, vol. 1, no. 3, pp. 339-372, 2009.

[20] Z. Liu and S. Peng, "Boundary processing of bidimensional emd using texture synthesis," IEEE Signal Processing Letters, vol. 12, no. 1, pp. 33-36, 2005.

[21] Z. Wu and N. E. Huang, "Ensemble empirical mode decomposition: A noise-assisted data analysis method," Advances in Adaptive Data Analysis, vol. 1, no. 1, pp. 1-41, 2009.

[22] C. Pozrikidis, Numerical Computation in Science and Engineering, 2nd ed. London, U.K.: Oxford University, 2008. 
[23] N. E. Huang, Z. Wu, S. R. Long, K. C. Arnold, X. Chen, and K. Blank, “On instantaneous frequency,” Advances in Adaptive Data Analysis, vol. 1, no. 2, pp. 177-229, 2009.

[24] R. C. Sharpley and V. Vatchev, "Analysis of the intrinsic mode functions," Constructive Approximation, vol. 24, no. 1, pp. 17-47, 2006.

[25] A. Nashif and D. Jones, Vibration Damping. Wiley-Interscience, 1985.

[26] "http://www.stat.unc.edu/faculty/hurd/stat185data/dataprog.html," May 5th 2009. 


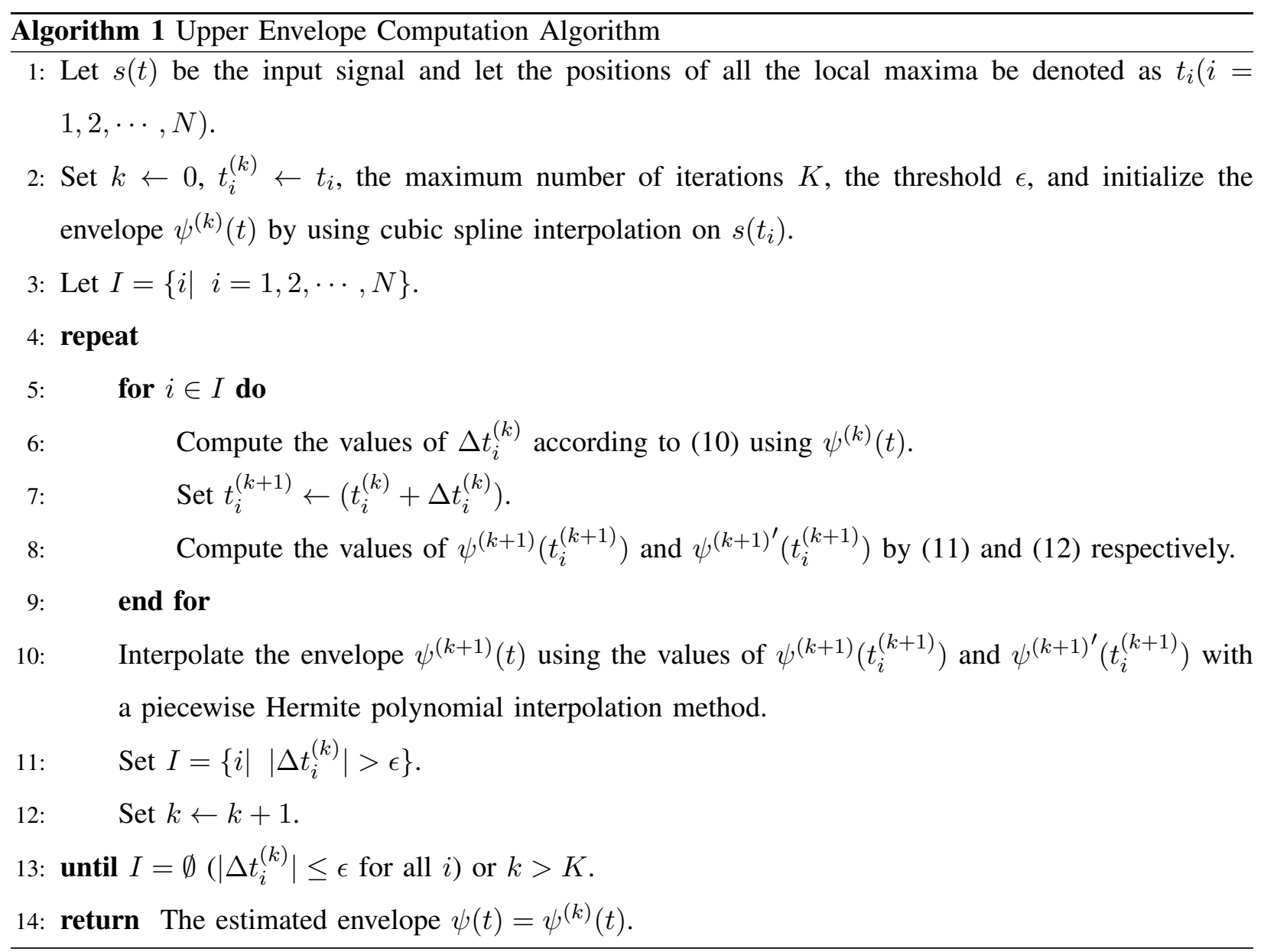

Remarks. 1. In step 2, we use cubic spline interpolation rather than Hermit polynomial interpolation because we only know the values of the extrema points at that point.

2. In the implementation of Step 9 of Algorithm 1, we do not need to interpolate the whole envelope because, according to (10), the value of $\Delta t_{i}^{(k)}$ only depends on the first and second derivatives of the signal and the envelope. Therefore, we only need to interpolate the values of points in the neighborhood of $t_{i}^{k+1}$. We also use a higher order piecewise Hermite interpolation method [22] to improve the smoothness of the estimated envelope. More specifically, we define the $k$ th order piecewise Hermite interpolation as follows:

$$
\begin{gathered}
P_{i-1}\left(t_{i}\right)=P_{i}\left(t_{i}\right)=s\left(t_{i}\right), \quad P_{i-1}^{\prime}\left(t_{i}\right)=P_{i}^{\prime}\left(t_{i}\right)=s^{\prime}\left(t_{i}\right), \\
P_{i-1}^{(j)}\left(t_{i}\right)=P_{i}^{(j)}\left(t_{i}\right), 2 \leq j \leq k
\end{gathered}
$$

where $P_{i}$ denotes the segment of the estimated envelope from $t_{i}$ to $t_{i+1}$, and $P_{i}^{(j)}\left(t_{i}\right)$ denotes the $j$ th derivatives of $P_{i}$ at $t_{i}$.

3. The stopping criterion $k>K$ is enforced because we cannot prove that the algorithm always stop when $I=\emptyset$ is obtained. In Appendix A, we derive the conditions for the algorithm to converge with 


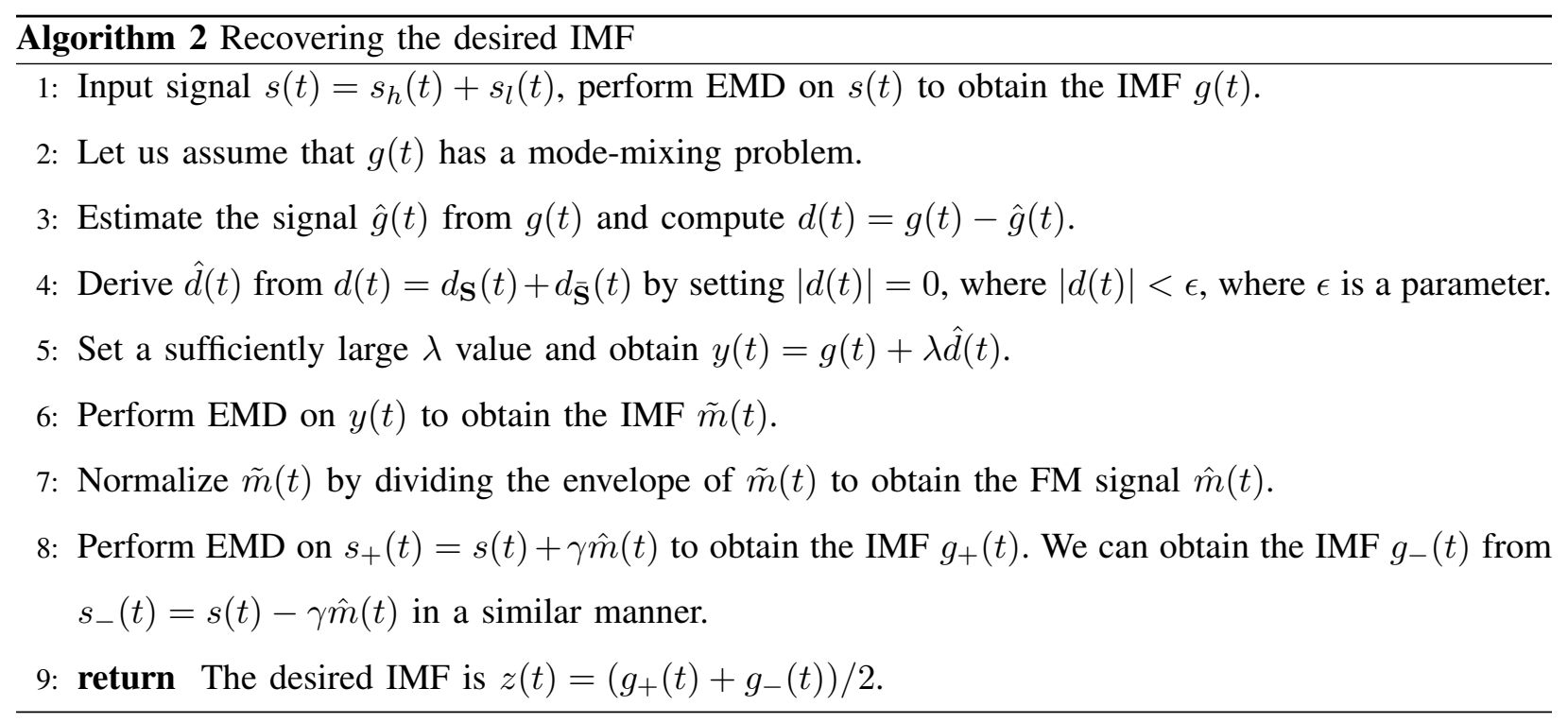

Remarks. 1. In Step 3, $\hat{g}(t)=\hat{a}(t) \cos (\hat{\phi}(t))$ is estimated as follows. We use Algorithm 1 to calculate the tangential points $\left\{\tau_{i}\right\}$ of $|g(t)|$, and derive the envelope $\hat{a}(t)$; then, we perform cubic spline interpolation on those tangential points by assuming that $\phi\left(\tau_{i}\right)=i \pi$.

2. In Step 4, we choose a threshold $\epsilon$ and let $\hat{d}(t)=0$ when $|d(t)|<\epsilon$ at any $t$. Our analysis in Section IV-A ensures that $\hat{d}(t)=d_{\mathbf{S}}(t)$ after Step 4.

3. In Step 7, we use Algorithm 1 to estimate the envelope of $|\tilde{m}(t)|$ and denote it as $\psi_{m}(t)$; and then derive the normalized masking signal $\hat{m}(t)=\tilde{m}(t) / \psi_{m}(t)$. 